\title{
Design and Use of Fluorogenic Aldehydes for Monitoring the Progress of Aldehyde Transformations
}

\author{
Fujie Tanaka,* Nobuyuki Mase, Carlos F. Barbas, III*
}

The Skaggs Institute for Chemical Biology and the Departments of Chemistry and Molecular Biology, The Scripps Research Institute, 10550 North Torrey Pines Road, La Jolla, California 92037

Corresponding author e-mail: carlos@scripps.edu, ftanaka@scripps.edu

\section{Supporting Information}

Fluorescence Spectra ----------------------------------------- S2

Graphs of Standards of $\mathbf{8}$ and $\mathbf{1 6}$--------------------------- S6

Synthesis and Characterization of Compounds ----------- S7

Hard copy of NMR ----------------------------------------- S11 
Fluorescence Spectra. Fuorescence was recorded on Spectra Max Gemini (Molecular Devices) using $100 \mu \mathrm{L}$ of a solution in a 96-well polypropyrene plate (Thomson Instrument Company 923175) at $26^{\circ} \mathrm{C}$. The data are shown after background correction.

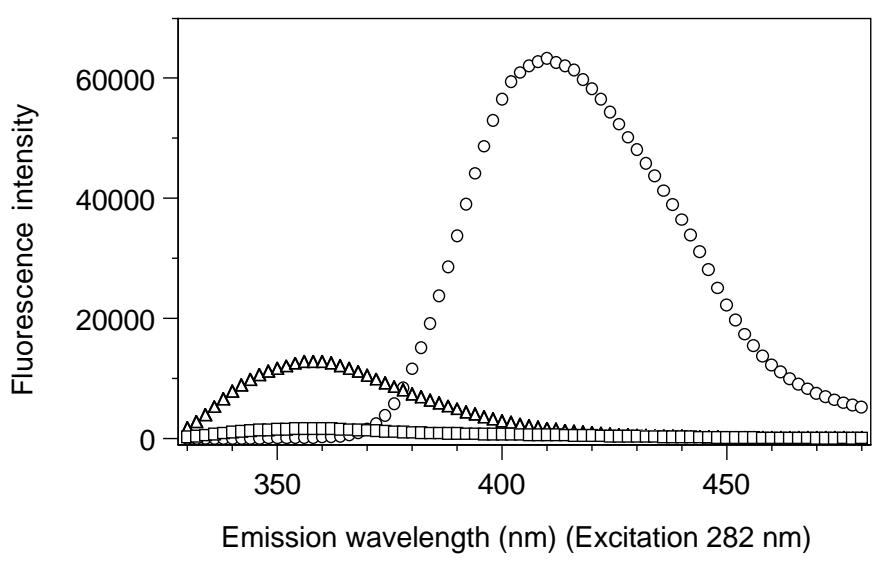

Figure S1. Fluorescence emission spectra ( $\lambda \operatorname{ex} 282 \mathrm{~nm}$ ) of 1, 2, and 2-aminonaphthalene in $0.5 \%$ $\mathrm{CH}_{3} \mathrm{CN}-0.5 \%$ 2-PrOH-99\% DMSO. Square, $1(50 \mu \mathrm{M})$; triangle, $2(50 \mu \mathrm{M})$; circle, 2 aminonaphthalene $(50 \mu \mathrm{M})$.

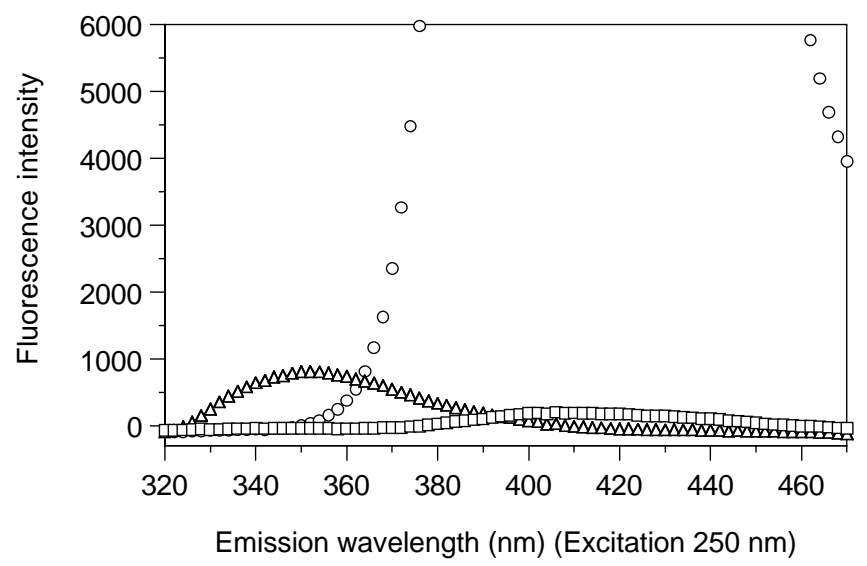

Figure S2. Fluorescence emission spectra ( $\lambda$ ex $250 \mathrm{~nm}$ ) of 1, 2, and 2-aminonaphthalene in $0.5 \%$ $\mathrm{CH}_{3} \mathrm{CN}-0.5 \%$ 2-PrOH-99\% (50 mM Na phosphate, $\mathrm{pH}$ 7.0). Square, 1 (50 $\left.\mu \mathrm{M}\right)$; triangle, 2 (50 $\mu \mathrm{M})$; circle, 2-aminonaphthalene $(50 \mu \mathrm{M})$. 


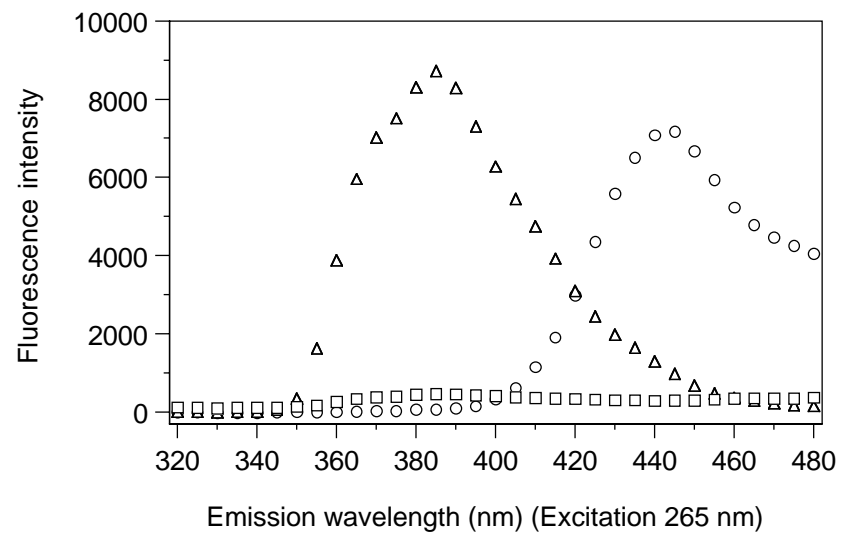

Figure S3. Fluorescence emission spectra $(\lambda \operatorname{ex} 265 \mathrm{~nm})$ of $\mathbf{7}, \mathbf{8}$, and $\mathbf{1 3}$ in $0.5 \% \mathrm{CH}_{3} \mathrm{CN}-0.5 \% 2-$ PrOH-99\% DMSO. Square, 7 (5 $\mu \mathrm{M})$; triangle, 8 (5 $\mu \mathrm{M})$; circle, 13 (5 $\mu \mathrm{M})$.

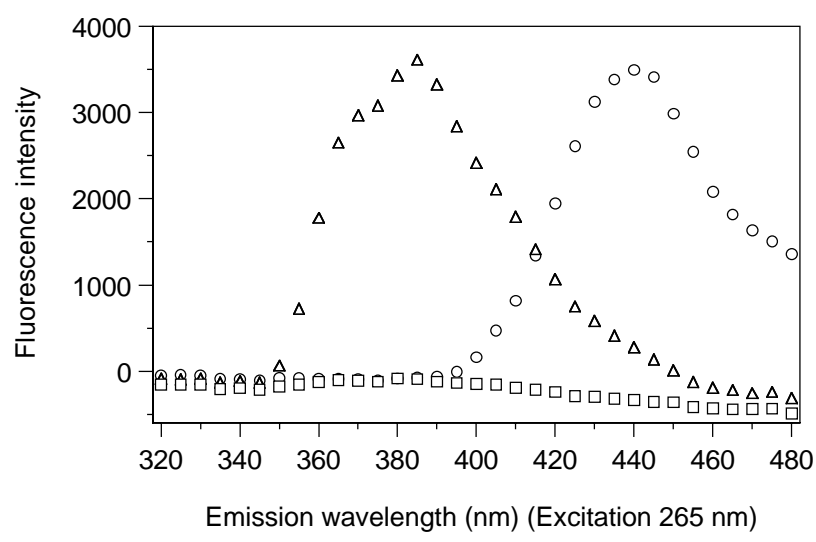

Figure S4. Fluorescence emission spectra $(\lambda \operatorname{ex} 265 \mathrm{~nm})$ of 7, 8, and 13 in $0.5 \% \mathrm{CH}_{3} \mathrm{CN}-0.5 \% 2$ PrOH-99\% DMF. Square, 7 (5 $\mu \mathrm{M})$; triangle, 8 (5 $\mu \mathrm{M})$; circle, 13 (5 $\mu \mathrm{M})$.

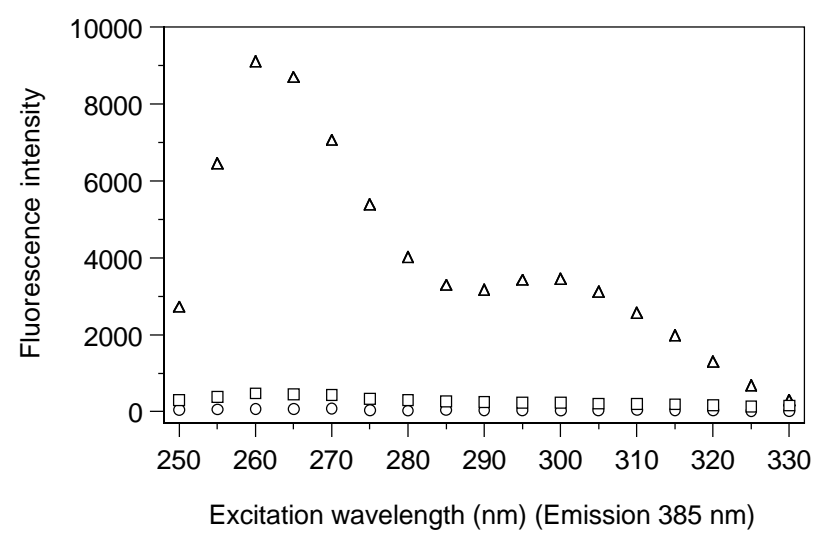

Figure S5. Fluorescence excitation spectra ( $\lambda \mathrm{em} 385 \mathrm{~nm}$ ) of 7, 8, and $\mathbf{1 3}$ in $0.5 \% \mathrm{CH}_{3} \mathrm{CN}-0.5 \% 2-$ PrOH-99\% DMSO. Square, 7 (5 $\mu \mathrm{M})$; triangle, 8 (5 $\mu \mathrm{M})$; circle, 13 (5 $\mu \mathrm{M})$. 


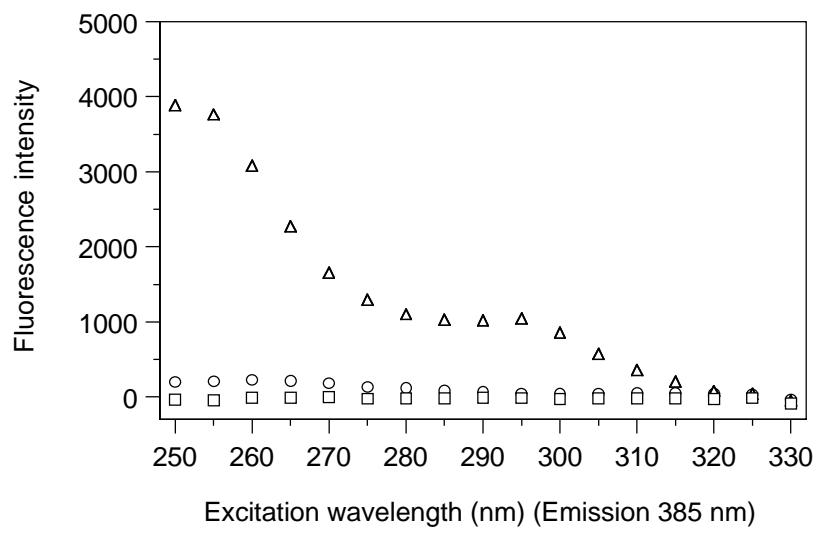

Figure S6. Fluorescence excitation spectra $\left(\lambda \mathrm{em} 385 \mathrm{~nm}\right.$ ) of 7, 8, and 13 in $0.5 \% \mathrm{CH}_{3} \mathrm{CN}-0.5 \% 2$ PrOH-99\% (50 mM Na phosphate, pH 7.0). Square, 7 (5 $\mu \mathrm{M})$ ) triangle, 8 (5 $\mu \mathrm{M})$; circle, 13 (5 $\mu \mathrm{M})$.

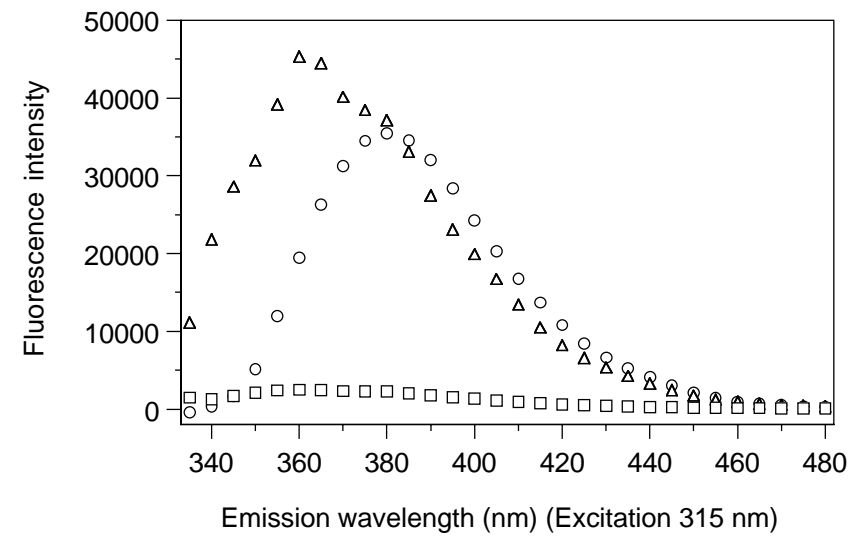

Figure S7. Fluorescence emission spectra $(\lambda \operatorname{ex} 315 \mathrm{~nm})$ of 9, 10, and 4-(1H-benzimidazol-2yl)aniline in 0.5\% $\mathrm{CH}_{3} \mathrm{CN}-0.5 \%$ 2-PrOH-99\% DMSO. Square, $9(5 \mu \mathrm{M})$; triangle, $10(5 \mu \mathrm{M})$; circle, 4 -(1H-benzimidazol-2-yl)aniline $(5 \mu \mathrm{M})$. 


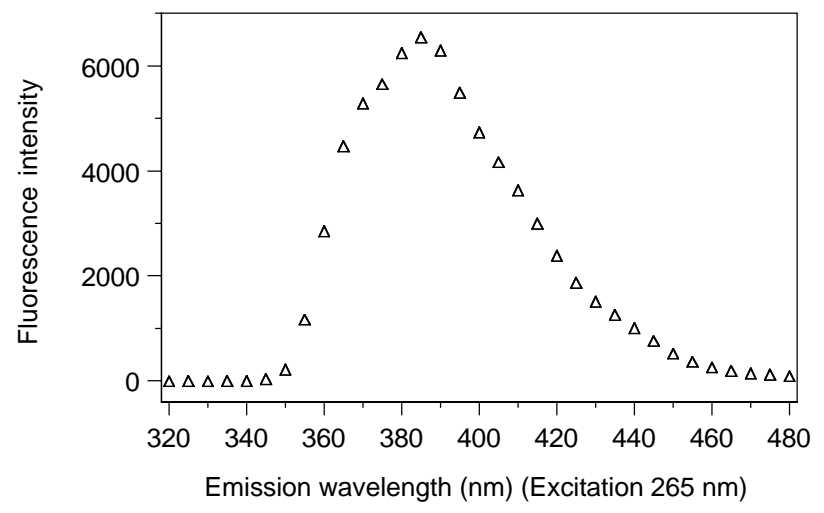

Figure S8. Fluorescence emission spectra $(\lambda \operatorname{ex} 265 \mathrm{~nm})$ of $\mathbf{1 4}(5 \mu \mathrm{M})$ in $0.5 \% \mathrm{CH}_{3} \mathrm{CN}-0.5 \% 2$ PrOH-99\% DMSO.

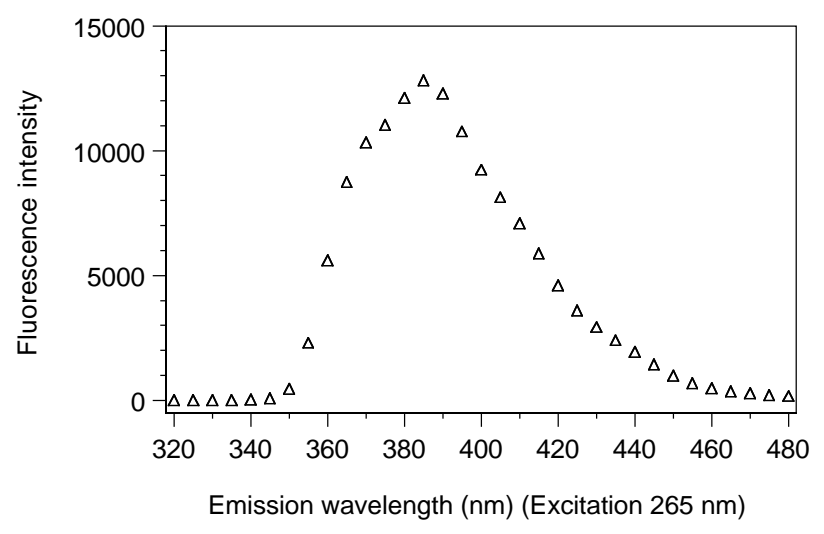

Figure S9. Fluorescence emission spectra $(\lambda \operatorname{ex} 265 \mathrm{~nm})$ of $15(5 \mu \mathrm{M})$ in $0.5 \% \mathrm{CH}_{3} \mathrm{CN}-0.5 \% 2$ PrOH-99\% DMSO.

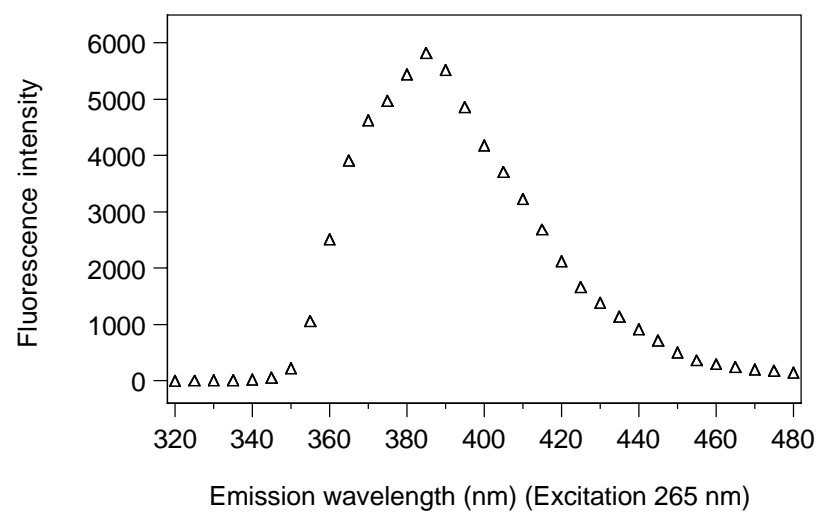

Figure S10. Fluorescence emission spectra $(\lambda \operatorname{ex} 265 \mathrm{~nm})$ of $\mathbf{1 6}(5 \mu \mathrm{M})$ in $0.5 \% \mathrm{CH}_{3} \mathrm{CN}-0.5 \% 2$ PrOH-99\% DMSO. 

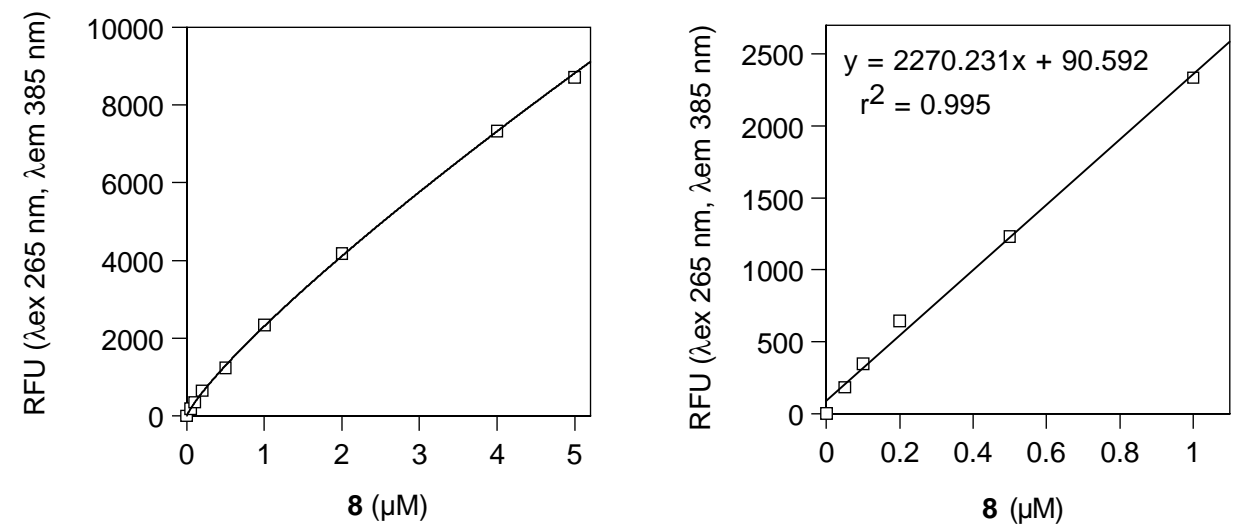

Figure S11. Standard of aldol 8 in $0.5 \% \mathrm{CH}_{3} \mathrm{CN}-0.5 \%$ 2-PrOH-99\% DMSO.

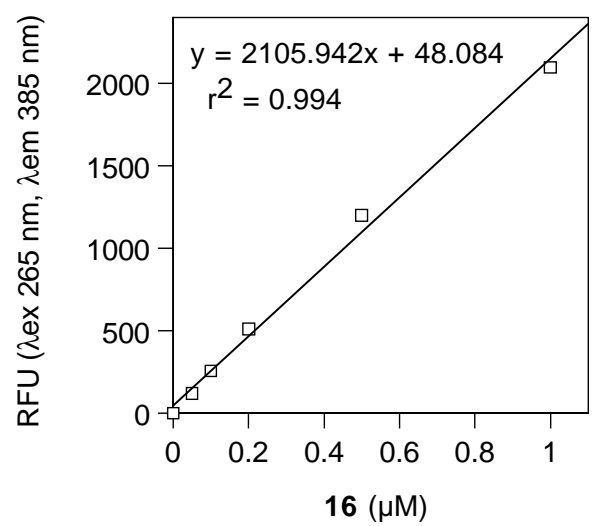

Figure S12. Standard of alcohol 16 in $0.5 \% \mathrm{CH}_{3} \mathrm{CN}-0.5 \%$ 2-PrOH-99\% DMSO. 
3-(4-Formylphenyl)- $N$-naphthalen-2-yl-propionamide (1). A mixture of 3-(4formylphenyl)propionic acid (70.0 mg, $0.393 \mathrm{mmol}), 2$-aminonaphthalene $(57.1 \mathrm{mg}, 0.399 \mathrm{mmol})$, 1-(3-dimethylaminopropyl)-3-ethylcarbodiimide hydrochloride (109.5 $\mathrm{mg}, 0.571 \mathrm{mmol}$ ), and DMAP $(1.0 \mathrm{mg}, 0.008 \mathrm{mmol})$ in $\mathrm{CH}_{2} \mathrm{Cl}_{2}(8.0 \mathrm{~mL})$ was stirred at room temperature for $2.5 \mathrm{~h}$. The reaction mixture was added to $\mathrm{H}_{2} \mathrm{O}$ and extracted with $\mathrm{CH}_{2} \mathrm{Cl}_{2}$. The organic layers were washed with brine, dried over $\mathrm{MgSO}_{4}$, filtered, concentrated in vacuo, and flash chromatographed $($ EtOAc/hexane $=2: 3)$ to afford $1(83.5 \mathrm{mg}, 70 \%) .{ }^{1} \mathrm{H}$ NMR $\left(400 \mathrm{MHz}, \mathrm{CDCl}_{3}\right): \delta 9.98(\mathrm{~s}, 1 \mathrm{H})$, $8.17(\mathrm{~s}, 1 \mathrm{H}), 7.84-7.77(\mathrm{~m}, 5 \mathrm{H}), 7.48-7.36(\mathrm{~m}, 5 \mathrm{H}), 7.22(\mathrm{~s}, 1 \mathrm{H}), 3.19(\mathrm{t}, J=7.6 \mathrm{~Hz}, 2 \mathrm{H}), 2.76(\mathrm{t}, J$ $=7.6 \mathrm{~Hz}, 2 \mathrm{H})$. MALDI-FTMS: calcd for $\mathrm{C}_{20} \mathrm{H}_{18} \mathrm{NO}_{2}\left(\mathrm{MH}^{+}\right)$304.1332, found 304.1333.

3-[4-(1-Hydroxy-3-oxobutyl)phenyl]- $N$-naphthalen-2-yl-propionamide (2). Aldol 2 was prepared by the proline-catalyzed aldol reaction of acetone and aldehyde $\mathbf{1}$ as described previously. ${ }^{\mathrm{S} 1}{ }^{1} \mathrm{H}$ NMR $\left(500 \mathrm{MHz}, \mathrm{CDCl}_{3}\right): \delta 8.15(\mathrm{~s}, 1 \mathrm{H}), 7.78-7.75(\mathrm{~m}, 3 \mathrm{H}), 7.47-7.35(\mathrm{~m}, 3 \mathrm{H})$, 7.30-7.22 (m, 5H), $5.12(\mathrm{ddd}, J=2.3 \mathrm{~Hz}, 2.6 \mathrm{~Hz}, 7.3 \mathrm{~Hz}, 1 \mathrm{H}), 3.27(\mathrm{~d}, J=2.3 \mathrm{~Hz}, 1 \mathrm{H}), 3.08(\mathrm{t}, J=$ $6.2 \mathrm{~Hz}, 2 \mathrm{H}), 2.87$ (dd, $J=7.3 \mathrm{~Hz}, 14.1 \mathrm{~Hz}, 1 \mathrm{H}), 2.79(\mathrm{dd}, J=2.6 \mathrm{~Hz}, 14.1 \mathrm{~Hz}, 1 \mathrm{H}), 2.70(\mathrm{t}, J=6.2$ $\mathrm{Hz}, 2 \mathrm{H}), 2.18(\mathrm{~s}, 3 \mathrm{H}) .{ }^{13} \mathrm{C} \mathrm{NMR}\left(100 \mathrm{MHz} \mathrm{CDCl}_{3}\right): \delta 209.2,170.4,140.8,140.1,135.1,133.8$, 130.6, 128.7, 128.6, 127.6, 127.5, 126.5, 126.0, 125.0, 119.7, 116.6, 69.6, 51.8, 39.4, 31.1, 30.7. MALDI-FTMS: calcd for $\mathrm{C}_{23} \mathrm{H}_{23} \mathrm{NO}_{3} \mathrm{Na}\left(\mathrm{MNa}^{+}\right)$384.1570, found 384.1579.

4-Formyl- $N$-naphthalen-2-yl-benzamide (3). ${ }^{1} \mathrm{H} \mathrm{NMR}\left(400 \mathrm{MHz}, \mathrm{CDCl}_{3}\right): \delta 10.1$ (s, 1H), 8.36 (brs, $1 \mathrm{H}), 8.10-8.00(\mathrm{~m}, 4 \mathrm{H}), 7.88-7.82(\mathrm{~m}, 3 \mathrm{H}), 7.60(\mathrm{dd}, J=2.0 \mathrm{~Hz}, 8.8 \mathrm{~Hz}, 1 \mathrm{H}), 7.53-$ 7.43 (m, 2H). MALDI-FTMS: calcd for $\mathrm{C}_{18} \mathrm{H}_{14} \mathrm{O}_{2} \mathrm{~N}\left(\mathrm{MH}^{+}\right)$276.1019, found 276.1022.

4-(1-Hydroxy-3-oxobutyl)- $N$-naphthalen-2-yl-benzamide (4). ${ }^{1} \mathrm{H}$ NMR (400 MHz, $\left.\mathrm{CDCl}_{3}-\mathrm{CD}_{3} \mathrm{OD}\right): \delta 9.21(\mathrm{~s}, 1 \mathrm{H} \times 0.7), 8.32(\mathrm{~s}, 1 \mathrm{H}), 7.90(\mathrm{~d}, J=8.2 \mathrm{~Hz}, 2 \mathrm{H}), 7.83-7.78(\mathrm{~m}, 3 \mathrm{H})$, $7.66(\mathrm{dd}, J=2.0 \mathrm{~Hz}, 8.8 \mathrm{~Hz}, 1 \mathrm{H}), 7.49-7.40(\mathrm{~m}, 2 \mathrm{H}), 7.46$ (d, $J=8.2 \mathrm{~Hz}, 2 \mathrm{H}), 5.19$ (dd, $J=3.8 \mathrm{~Hz}$, $9.1 \mathrm{~Hz}, 1 \mathrm{H}), 2.99$ (s, 1H), $2.91(\mathrm{dd}, J=8.9 \mathrm{~Hz}, 16.7 \mathrm{~Hz}, 1 \mathrm{H}), 2.79$ (dd, J=3.7 Hz, $16.7 \mathrm{~Hz}, 1 \mathrm{H})$, $2.21(\mathrm{~s}, 3 \mathrm{H})$. MALDI-FTMS: calcd for $\mathrm{C}_{21} \mathrm{H}_{20} \mathrm{NO}_{3}\left(\mathrm{MH}^{+}\right)$334.1438, found 334.1440.

3-(4-Formylphenyl)- $N$-naphthalen-1-yl-propionamide (5). ${ }^{1} \mathrm{H}$ NMR (400 MHz, $\left.\mathrm{CDCl}_{3}-\mathrm{CD}_{3} \mathrm{OD}\right): \delta 9.98(\mathrm{~s}, 1 \mathrm{H}), 7.86-7.83(\mathrm{~m}, 3 \mathrm{H}), 7.71(\mathrm{~d}, J=8.2 \mathrm{~Hz}, 1 \mathrm{H}), 7.67(\mathrm{~d}, J=7.3 \mathrm{~Hz}$, 
1H), $7.57(\mathrm{~d}, J=8.2 \mathrm{~Hz}, 1 \mathrm{H}), 7.50-7.38(\mathrm{~m}, 5 \mathrm{H}), 3.20(\mathrm{t}, J=7.6 \mathrm{~Hz}, 2 \mathrm{H}), 2.87(\mathrm{t}, J=7.6 \mathrm{~Hz}, 2 \mathrm{H})$.

MALDI-FTMS: calcd for $\mathrm{C}_{20} \mathrm{H}_{18} \mathrm{NO}_{2}\left(\mathrm{MH}^{+}\right)$304.1332, found 304.1331.

3-[4-(1-Hydroxy-3-oxobutyl)-phenyl]- $N$-naphthalen-1-yl-propionamide $\quad(6) . \quad{ }^{1} \mathrm{H}$ NMR (400 MHz, $\left.\mathrm{CDCl}_{3}-\mathrm{CD}_{3} \mathrm{OD}\right): \delta 7.85(\mathrm{~m}, 1 \mathrm{H})$, 7.75-7.68 (m, 2H), $7.57(\mathrm{~m}, 1 \mathrm{H})$, 7.49-7.43 (m, 3H), 7.33-7.27 (m, 4H), $5.13(\mathrm{dd}, J=3.2 \mathrm{~Hz}, 9.1 \mathrm{~Hz}, 1 \mathrm{H}), 3.11(\mathrm{t}, J=7.6 \mathrm{~Hz}, 2 \mathrm{H}), 2.89(\mathrm{dd}, J=$ $9.1 \mathrm{~Hz}, 17 \mathrm{~Hz}, 1 \mathrm{H}), 2.80$ (t, $J=7.6 \mathrm{~Hz}, 2 \mathrm{H}), 2.77$ (dd, J = 3.2 Hz, $17 \mathrm{~Hz}, 1 \mathrm{H}), 2.19$ (s, 3H). MALDI-FTMS: calcd for $\mathrm{C}_{23} \mathrm{H}_{23} \mathrm{NO}_{3} \mathrm{Na}\left(\mathrm{MNa}^{+}\right)$384.1570, found 384.1578.

3-(4-Formylphenyl)- $N$-phenanthren-9-yl-propionamide (7). ${ }^{1} \mathrm{H}$ NMR (400 MHz, $\left.\mathrm{CDCl}_{3}-\mathrm{CD}_{3} \mathrm{OD}\right): \delta 9.99(\mathrm{~s}, 1 \mathrm{H}), 8.71(\mathrm{~d}, J=8.8 \mathrm{~Hz}, 1 \mathrm{H}), 8.63(\mathrm{~d}, J=7.9 \mathrm{~Hz}, 1 \mathrm{H}), 8.03(\mathrm{~s}, 1 \mathrm{H})$, 7.88-7.84 (m, 3H), 7.67-7.49 (m, 7H), $3.24(\mathrm{t}, J=7.6 \mathrm{~Hz}, 2 \mathrm{H}), 2.91(\mathrm{t}, J=7.6 \mathrm{~Hz}, 2 \mathrm{H}) .{ }^{13} \mathrm{C} \mathrm{NMR}$ (100 MHz, $\left.\mathrm{CDCl}_{3}-\mathrm{CD}_{3} \mathrm{OD}\right): \delta 192.5,171.8,148.3,134.5,131.2,130.8,130.2,130.0,129.1,128.8$, 128.3, 127.5, 126.7, 126.5, 126.3, 122.8, 122.5, 122.2, 121.9, 37.6, 31.6. MALDI-FTMS: calcd for $\mathrm{C}_{24} \mathrm{H}_{20} \mathrm{NO}_{2}\left(\mathrm{MH}^{+}\right)$354.1488, found 354.1488.

3-[4-(1-Hydroxy-3-oxobutyl)phenyl]- $N$-phenanthren-9-yl-propionamide $\quad(8) . \quad{ }^{1} \mathrm{H}$ NMR (500 MHz, $\left.\mathrm{CDCl}_{3}\right): \delta 8.69(\mathrm{~d}, J=8.1 \mathrm{~Hz}, 1 \mathrm{H}), 8.60(\mathrm{~d}, J=7.7 \mathrm{~Hz}, 1 \mathrm{H}), 8.13(\mathrm{~s}, 1 \mathrm{H}), 7.83$ $(\mathrm{d}, J=7.3 \mathrm{~Hz}, 1 \mathrm{H}), 7.66-7.53(\mathrm{~m}, 5 \mathrm{H}), 7.38(\mathrm{~s}, 1 \mathrm{H}), 7.34-7.27(\mathrm{~m}, 4 \mathrm{H}), 5.14(\mathrm{~m} \mathrm{1H}), 3.30(1 \mathrm{H})$, $3.15(\mathrm{t}, J=7.6 \mathrm{~Hz}, 2 \mathrm{H}), 2.88-2.75(\mathrm{~m}, 2 \mathrm{H}), 2.84(\mathrm{t}, J=7.6 \mathrm{~Hz}, 2 \mathrm{H}), 2.17(\mathrm{~s}, 3 \mathrm{H}) \cdot{ }^{13} \mathrm{C}$ NMR $(100$ $\left.\mathrm{MHz}, \mathrm{CDCl}_{3}\right): \delta 209.2,171.0,140.9,140.1,131.6,131.0,130.0,128.7,128.6,127.0,126.9,126.7$, 126.3, 126.1, 123.3, 122.3, 121.2, 121.1, 69.6, 51.8, 39.3, 31.4, 30.7. MALDI-FTMS: calcd for $\mathrm{C}_{27} \mathrm{H}_{25} \mathrm{NO}_{3} \mathrm{Na}\left(\mathrm{MNa}^{+}\right)$434.1727, found 434.1732.

$N$-[4-(1H-Benzoimidazol-2-yl)phenyl]-3-(4-formylphenyl)-propionamide $\quad(9) .{ }^{1} \mathrm{H}$ NMR (400 MHz, $\left.\mathrm{CDCl}_{3}-\mathrm{CD}_{3} \mathrm{OD}\right): \delta 9.95(\mathrm{~s}, 1 \mathrm{H}), 8.01(\mathrm{~d}, J=8.5 \mathrm{~Hz}, 2 \mathrm{H}), 7.83(\mathrm{~d}, J=7.8 \mathrm{~Hz}$, 2H), $7.69(\mathrm{~d}, J=8.5 \mathrm{~Hz}, 2 \mathrm{H}), 7.62-7.60(\mathrm{~m}, 2 \mathrm{H}), 7.46(\mathrm{~d}, J=7.8 \mathrm{~Hz}, 2 \mathrm{H}), 7.28-7.25(\mathrm{~m}, 2 \mathrm{H}), 3.14$ $(\mathrm{t}, J=7.6 \mathrm{~Hz}, 2 \mathrm{H}), 2.76(\mathrm{t}, J=7.6 \mathrm{~Hz}, 2 \mathrm{H})$. MALDI-FTMS: calcd for $\mathrm{C}_{23} \mathrm{H}_{20} \mathrm{~N}_{3} \mathrm{O}_{2}\left(\mathrm{MH}^{+}\right)$ 370.1550 , found 370.1548 .

$N$-[4-(1H-Benzoimidazol-2-yl)phenyl]-3-(4-formylphenyl)-propionamide $(10) .{ }^{1} \mathrm{H}$ NMR (400 MHz, $\left.\mathrm{CDCl}_{3}-\mathrm{CD}_{3} \mathrm{OD}\right): \delta 8.01(\mathrm{~d}, J=8.1 \mathrm{~Hz}, 2 \mathrm{H}), 7.68(\mathrm{~d}, J=8.1 \mathrm{~Hz}, 2 \mathrm{H}), 7.65-7.56$ $(\mathrm{m}, 2 \mathrm{H}), 7.30-7.23(\mathrm{~m}, 6 \mathrm{H}), 5.10(\mathrm{~m}, 1 \mathrm{H}), 3.04(\mathrm{t}, J=8.0 \mathrm{~Hz}, 2 \mathrm{H}), 2.93(\mathrm{~m}, 1 \mathrm{H}), 2.76(\mathrm{~m}, 1 \mathrm{H}), 2,70$ 
(t, $J=8.0 \mathrm{~Hz}, 2 \mathrm{H}), 2.19$ (s, 3H). MALDI-FTMS: calcd for $\mathrm{C}_{26} \mathrm{H}_{26} \mathrm{~N}_{3} \mathrm{O}_{3}\left(\mathrm{MH}^{+}\right)$428.1969, found 428.1978 .

$N$-Fluoranthen-3-yl-3-(4-formylphenyl)-propionamide (11). ${ }^{1} \mathrm{H}$ NMR (400 MHz, $\left.\mathrm{CDCl}_{3}-\mathrm{CD}_{3} \mathrm{OD}\right): \delta 9.96(\mathrm{~s}, 1 \mathrm{H}), 7.99-7.35(\mathrm{~m}, 13 \mathrm{H}), 3.21(\mathrm{t}, J=7.6 \mathrm{~Hz}, 2 \mathrm{H}), 2.89(\mathrm{t}, J=7.6 \mathrm{~Hz}$, 2H). MALDI-FTMS: calcd for $\mathrm{C}_{26} \mathrm{H}_{20} \mathrm{NO}_{2}\left(\mathrm{MH}^{+}\right)$378.1488, found 378.1485.

$N$-Fluoranthen-3-yl-3-[4-(1-hydroxy-3-oxobutyl)phenyl]-propionamide $\quad(12) .{ }^{1} \mathrm{H}$ NMR (400 MHz, $\left.\mathrm{CDCl}_{3}-\mathrm{CD}_{3} \mathrm{OD}\right): \delta 8.01-7.28(\mathrm{~m}, 13 \mathrm{H}), 5.12(\mathrm{dd}, J=3.5 \mathrm{~Hz}, 9.1 \mathrm{~Hz}, 1 \mathrm{H}), 3.12$ $(\mathrm{t}, J=7.3 \mathrm{~Hz}, 2 \mathrm{H}), 2.91(\mathrm{dd}, J=9.1 \mathrm{~Hz}, 16.6 \mathrm{~Hz}, 1 \mathrm{H}), 2.86(\mathrm{t}, J=7.3 \mathrm{~Hz}, 2 \mathrm{H}), 2.76(\mathrm{dd}, J=3.5$ $\mathrm{Hz}, 16.6 \mathrm{~Hz}, 1 \mathrm{H}), 2.19$ (s, 3H). MALDI-FTMS: calcd for $\mathrm{C}_{29} \mathrm{H}_{25} \mathrm{NO}_{3}\left(\mathrm{M}^{+}\right)$435.1834, found 435.1830 .

3-[4-(1,2-Dihydroxy-3-oxobutyl)phenyl]- $N$-phenanthren-9-yl-propionamide (14). ${ }^{1} \mathrm{H}$ NMR (300 MHz, $\left.\mathrm{CDCl}_{3}\right) \delta 8.69(\mathrm{~d}, J=8.0 \mathrm{~Hz}, 1 \mathrm{H}), 8.60(\mathrm{~d}, J=7.4 \mathrm{~Hz}, 1 \mathrm{H}), 8.10$ (brs, $\left.1 \mathrm{H}\right)$, 7.90-7.80 (m, 1H), 7.78-7.45 (m, 5H), 7.45-7.25 (m, 4H), 5.10-4.85 (m, 1H), 4.50-4.25 (m, 1H), 3.80-2.72 (m, 6H), $2.22\left(\mathrm{~s}, 3 \mathrm{H}\right.$ x 1/4), 1.96 (s, 3H x 3/4). MALDI-FTMS calcd for $\mathrm{C}_{27} \mathrm{H}_{25} \mathrm{NO}_{4}$ $\left(\mathrm{MNa}^{+}\right): 450.1676$, found: 450.1677.

3-[4-(1-Hydroxybut-3-enyl)phenyl]- $N$-phenanthren-9-yl-propionamide (15). A mixture of aldehyde 7 (9.3 mg, $0.026 \mathrm{mmol})$, allylbromide (50 $\mu \mathrm{L}, 0.58 \mathrm{mmol})$, In $(9.1 \mathrm{mg}, 0.079$ $\mathrm{mmol})$ in $\mathrm{DMF}(0.4 \mathrm{~mL})-\mathrm{H}_{2} \mathrm{O}(0.05 \mathrm{~mL})$ was stirred at room temperature for $1.5 \mathrm{~h} .{ }^{\mathrm{S} 2}$ The reaction mixture was added to sat- $\mathrm{NH}_{4} \mathrm{Cl}$ and extracted with EtOAc. The organic layers were washed with brine, dried over $\mathrm{MgSO}_{4}$, filtered, concentrated in vacuo, and flash chromatographed $($ EtOAc/hexane $=2: 3)$ to afford $15(10.0 \mathrm{mg}, 96 \%)$. ${ }^{1} \mathrm{H}$ NMR $\left(500 \mathrm{MHz}, \mathrm{CDCl}_{3}\right): \delta 8.72(\mathrm{~d}, J=$ $8.1 \mathrm{~Hz}, 1 \mathrm{H}), 8.63(\mathrm{~d}, J=8.1 \mathrm{~Hz}, 1 \mathrm{H}), 8.19(\mathrm{~s}, 1 \mathrm{H}), 7.87(\mathrm{~d}, J=7.4 \mathrm{~Hz}, 1 \mathrm{H}), 7.70-7.55(\mathrm{~m}, 6 \mathrm{H})$, 7.37-7.30 (m, 4H), $5.80(\mathrm{~m}, 1 \mathrm{H}), 5.20-5.12(\mathrm{~m}, 2 \mathrm{H}), 4.75(\mathrm{~m}, 1 \mathrm{H}), 3.18(\mathrm{t}, J=7.4 \mathrm{~Hz}, 2 \mathrm{H}), 2.88(\mathrm{t}$, $J=7.4 \mathrm{~Hz}, 2 \mathrm{H}), 2.55-2.42$ (m, 2H), 2.00 (brs, 1H). ${ }^{13} \mathrm{C}$ NMR (100 MHz, $\mathrm{CDCl}_{3}$ ): $\delta 171.1,142.1$, $139.8,134.4,131.6,131.0,130.0,128.6,128.5,126.9,126.7,126.3,126.2,123.3,122.3,121.3$, 121.2, 118.5, 73.0, 43.8, 39.3, 31.4. MALDI-FTMS calcd for $\mathrm{C}_{27} \mathrm{H}_{25} \mathrm{NO}_{2} \mathrm{Na}\left(\mathrm{MNa}^{+}\right)$: 418.1777, found: 418.1777 . 
3-(4-Hydroxymethylphenyl)- $N$-phenanthren-9-yl-propionamide (16). A mixture of aldehyde 7 (10.0 mg, $0.028 \mathrm{mmol})$ and $\mathrm{NaBH}_{3} \mathrm{CN}(6.0 \mathrm{mg}, 0.095 \mathrm{mmol})$ in THF (0.5 mL)-2PrOH (0.1 mL)-50 mM Na phosphate, $\mathrm{pH} 7.0(0.2 \mathrm{~mL})$ was stirred at room temperature for 1 day. The reaction mixture was added to $s a t-\mathrm{NH}_{4} \mathrm{Cl}$ and extracted with EtOAc. The organic layers were washed with brine, dried over $\mathrm{MgSO}_{4}$, filtered, concentrated in vacuo, and flash chromatographed $($ EtOAc/hexane $=1: 1.2)$ to afford $16(4.3 \mathrm{mg}, 43 \%) .{ }^{1} \mathrm{H}$ NMR $\left(500 \mathrm{MHz}, \mathrm{CDCl}_{3}-\mathrm{CD}_{3} \mathrm{OD}\right): \delta$ $8.72(\mathrm{~d}, J=8.1 \mathrm{~Hz}, 1 \mathrm{H}), 8.65(\mathrm{~d}, J=7.7 \mathrm{~Hz}, 1 \mathrm{H}), 7.90(\mathrm{~s}, 1 \mathrm{H}), 7.86(\mathrm{~d}, J=7.0 \mathrm{~Hz}, 1 \mathrm{H}), 7.71-7.55$ (m, 5H), $7.35(\mathrm{~d}, J=8.5 \mathrm{~Hz}, 2 \mathrm{H}), 7.33(\mathrm{~d}, J=8.5 \mathrm{~Hz}, 2 \mathrm{H}), 4.65(\mathrm{~s}, 2 \mathrm{H}), 3.14(\mathrm{t}, J=7.5 \mathrm{~Hz}, 2 \mathrm{H})$, $2.88(\mathrm{t}, J=7.5 \mathrm{~Hz}, 2 \mathrm{H})$. MALDI-FTMS: calcd for $\mathrm{C}_{24} \mathrm{H}_{21} \mathrm{NO}_{2} \mathrm{Na}\left(\mathrm{MNa}^{+}\right)$378.1464, found 378.1468 .

\section{References}

(S1) Sakthivel, K.; Notz, W.; Bui, T.; Barbas, C. F., III J. Am. Chem. Soc. 2001, 123, 5260. (S2) Chan, T. H.; Yang, Y. J. Am. Chem. Soc. 1999, 121, 3228. 
Compound 1, ${ }^{1} \mathrm{H}$ NMR $\left(400 \mathrm{MHz}, \mathrm{CDCl}_{3}\right)$

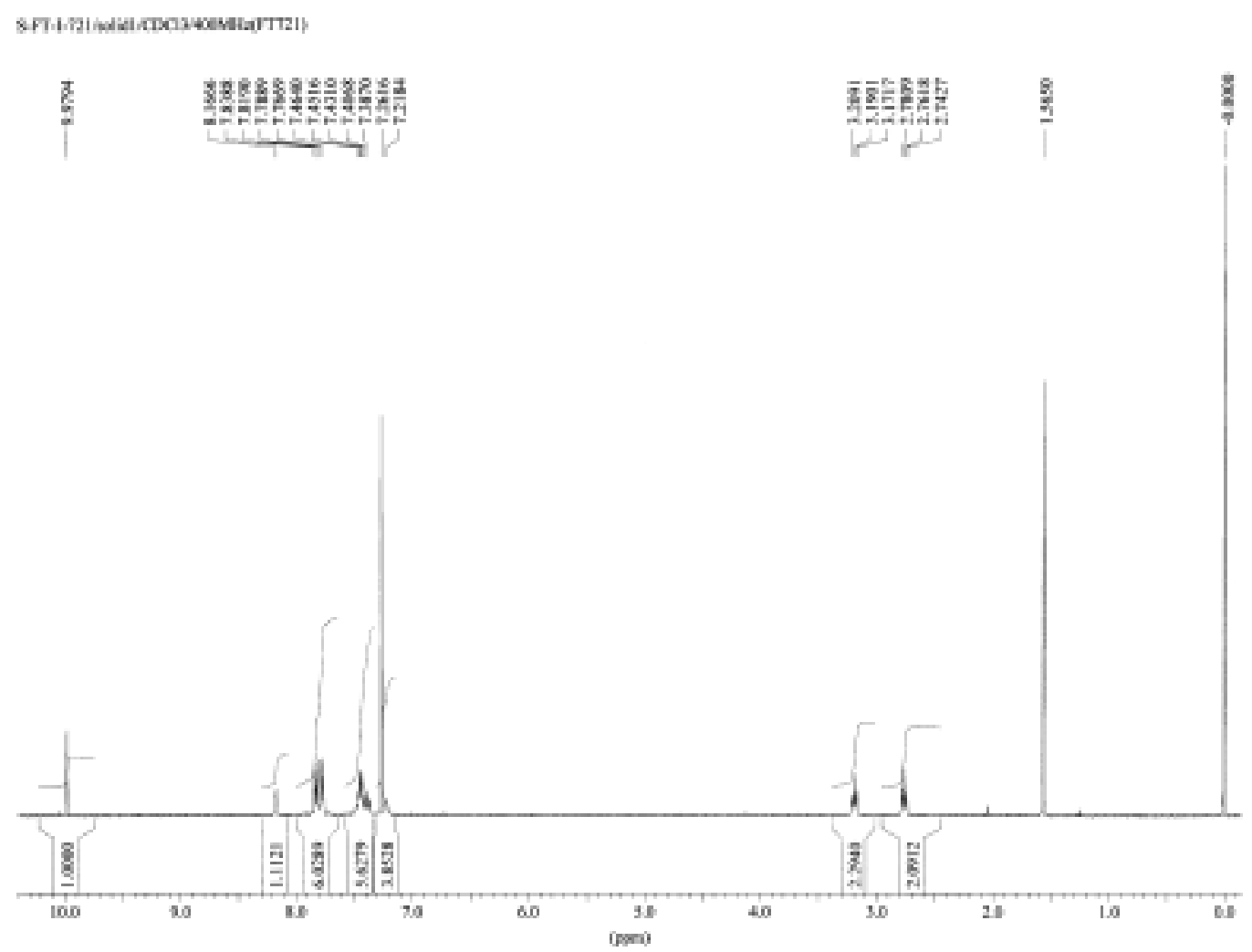

Compound 2, ${ }^{1} \mathrm{H} \mathrm{NMR}\left(500 \mathrm{MHz}, \mathrm{CDCl}_{3}\right)$

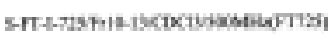

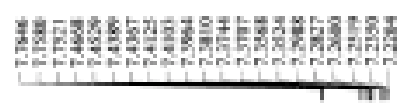

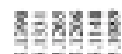

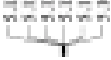

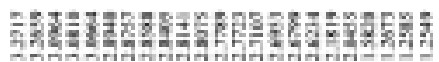

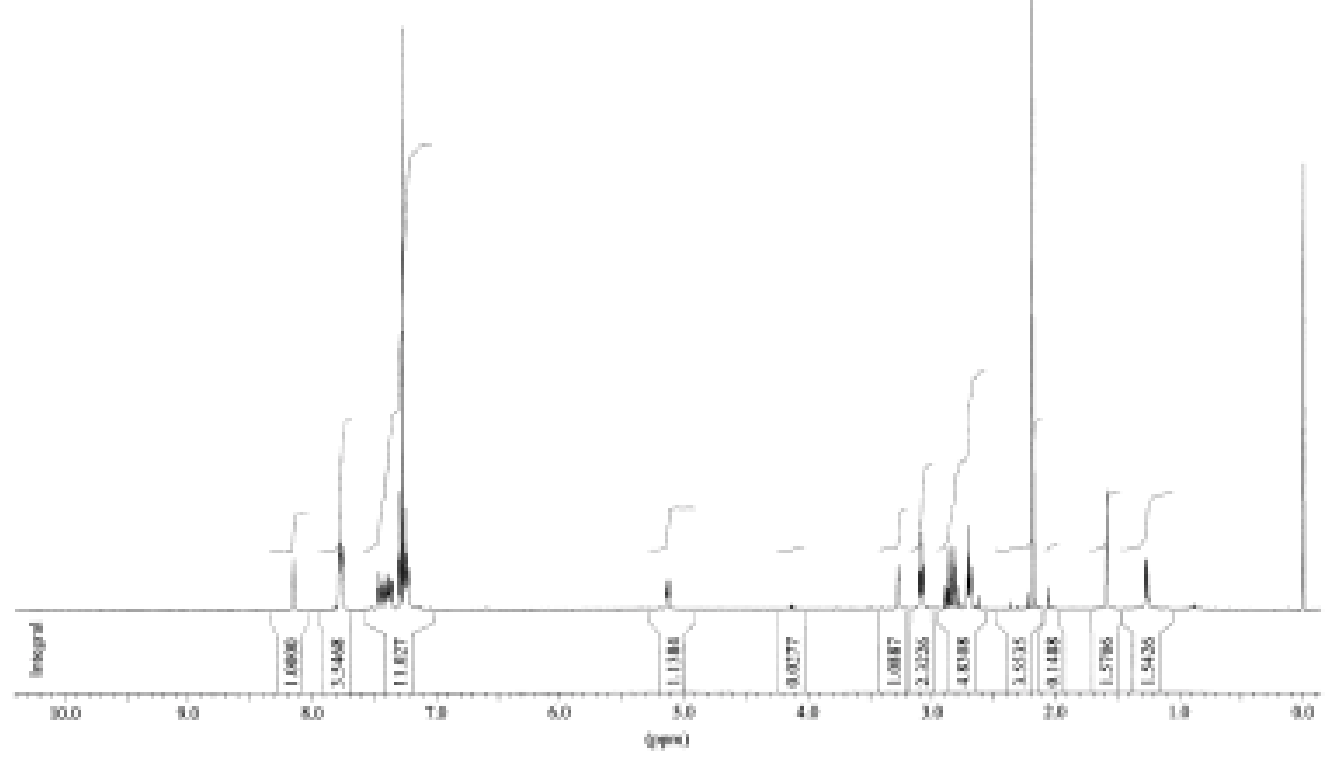


Compound 2, ${ }^{13} \mathrm{C}$ NMR $\left(100 \mathrm{MHz}, \mathrm{CDCl}_{3}\right)$

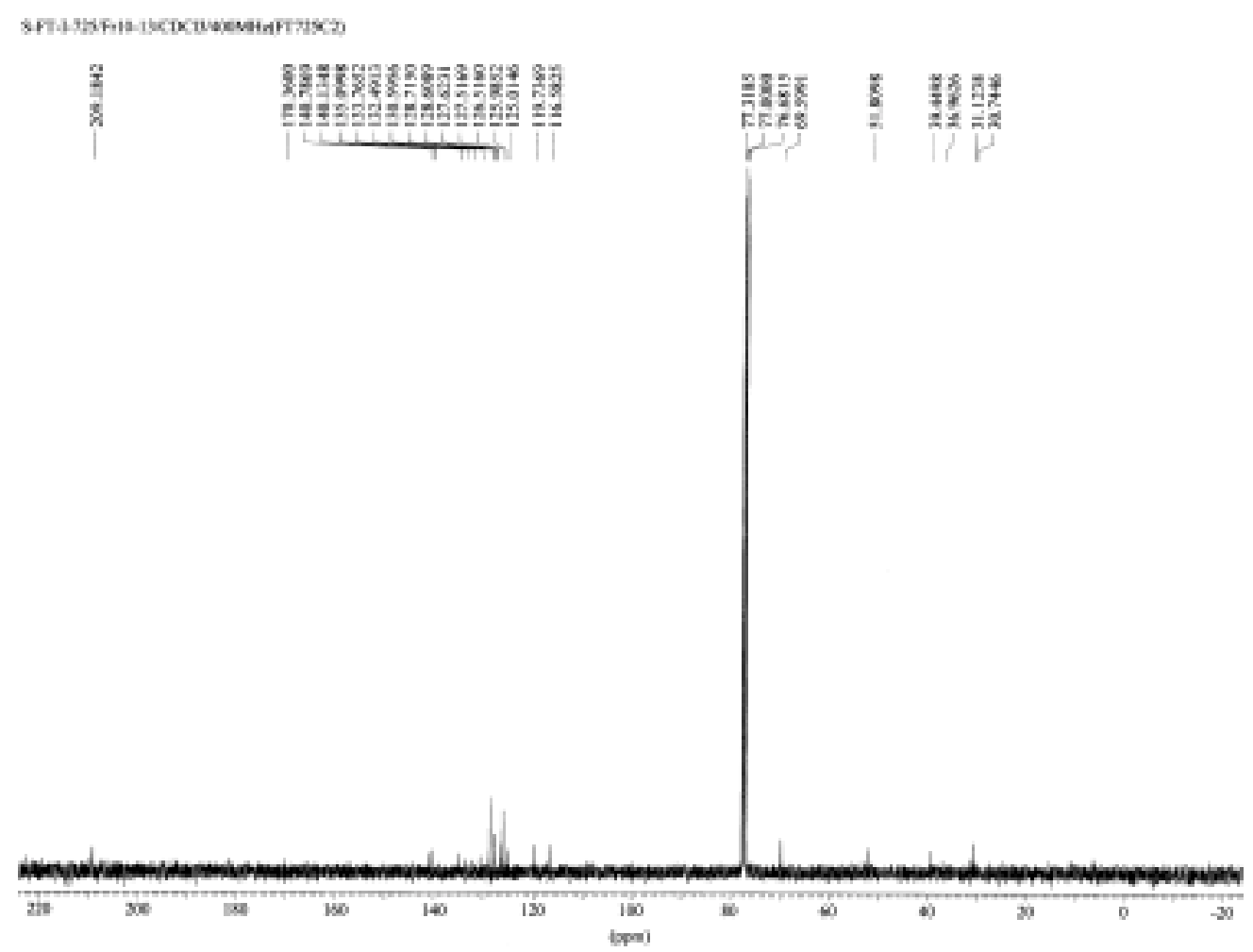

Compound 3, ${ }^{1} \mathrm{H}$ NMR $\left(400 \mathrm{MHz}, \mathrm{CDCl}_{3}\right)$
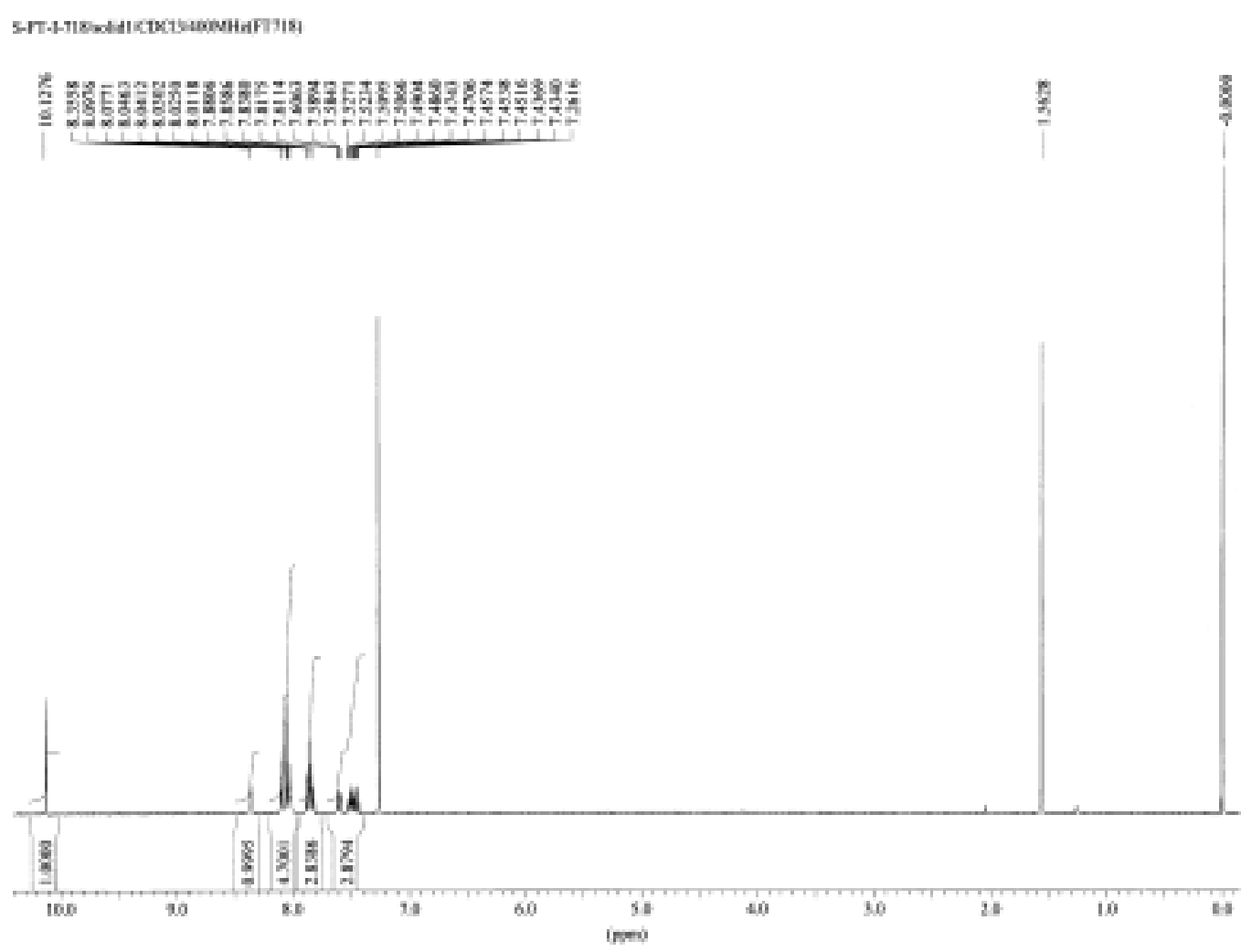
Compound 4, ${ }^{1} \mathrm{H} \mathrm{NMR}\left(400 \mathrm{MHz}, \mathrm{CDCl}_{3}-\mathrm{CD}_{3} \mathrm{OD}\right)$
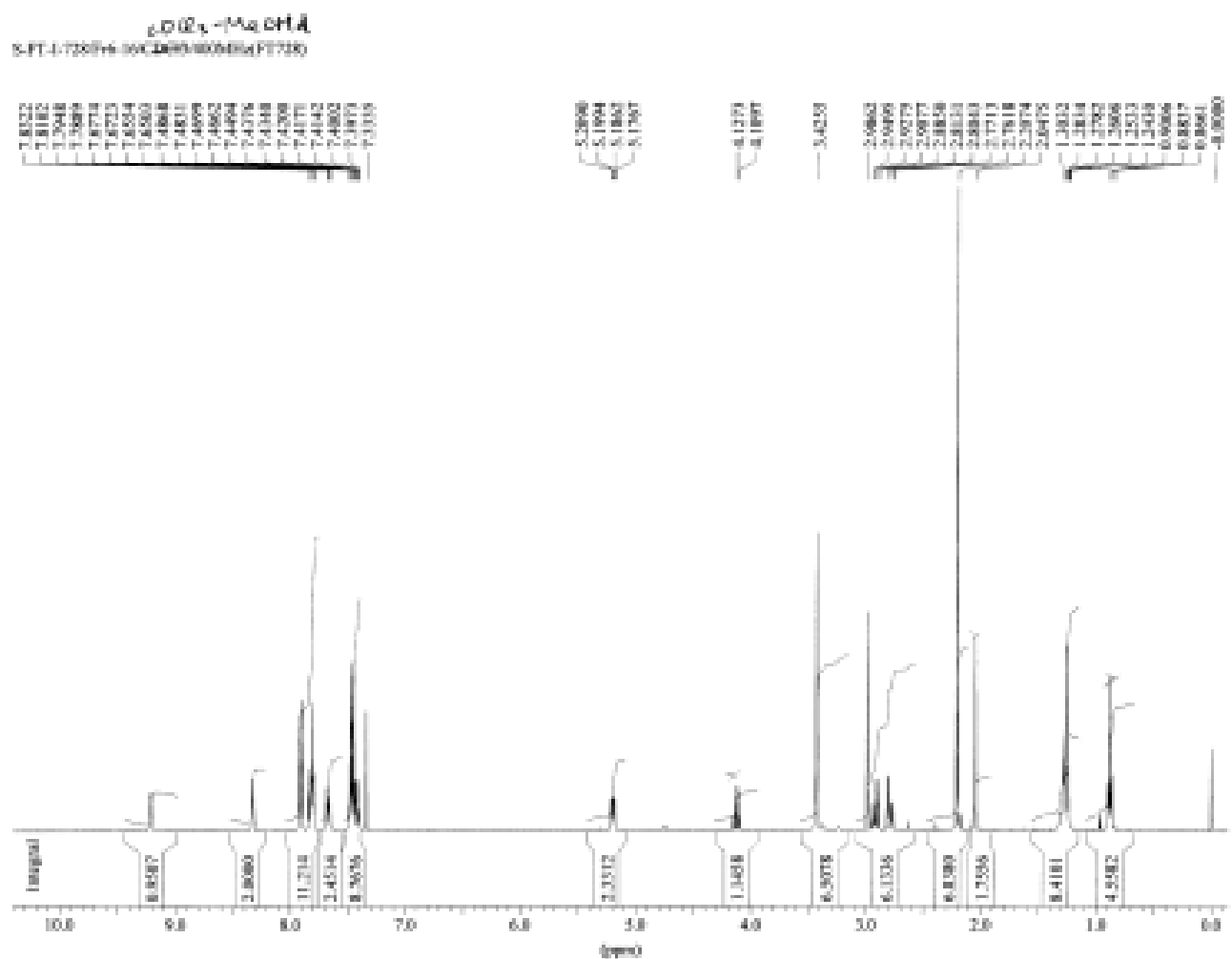

Compound 5, ${ }^{1} \mathrm{H}$ NMR $\left(400 \mathrm{MHz}, \mathrm{CDCl}_{3}-\mathrm{CD}_{3} \mathrm{OD}\right)$

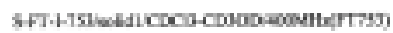
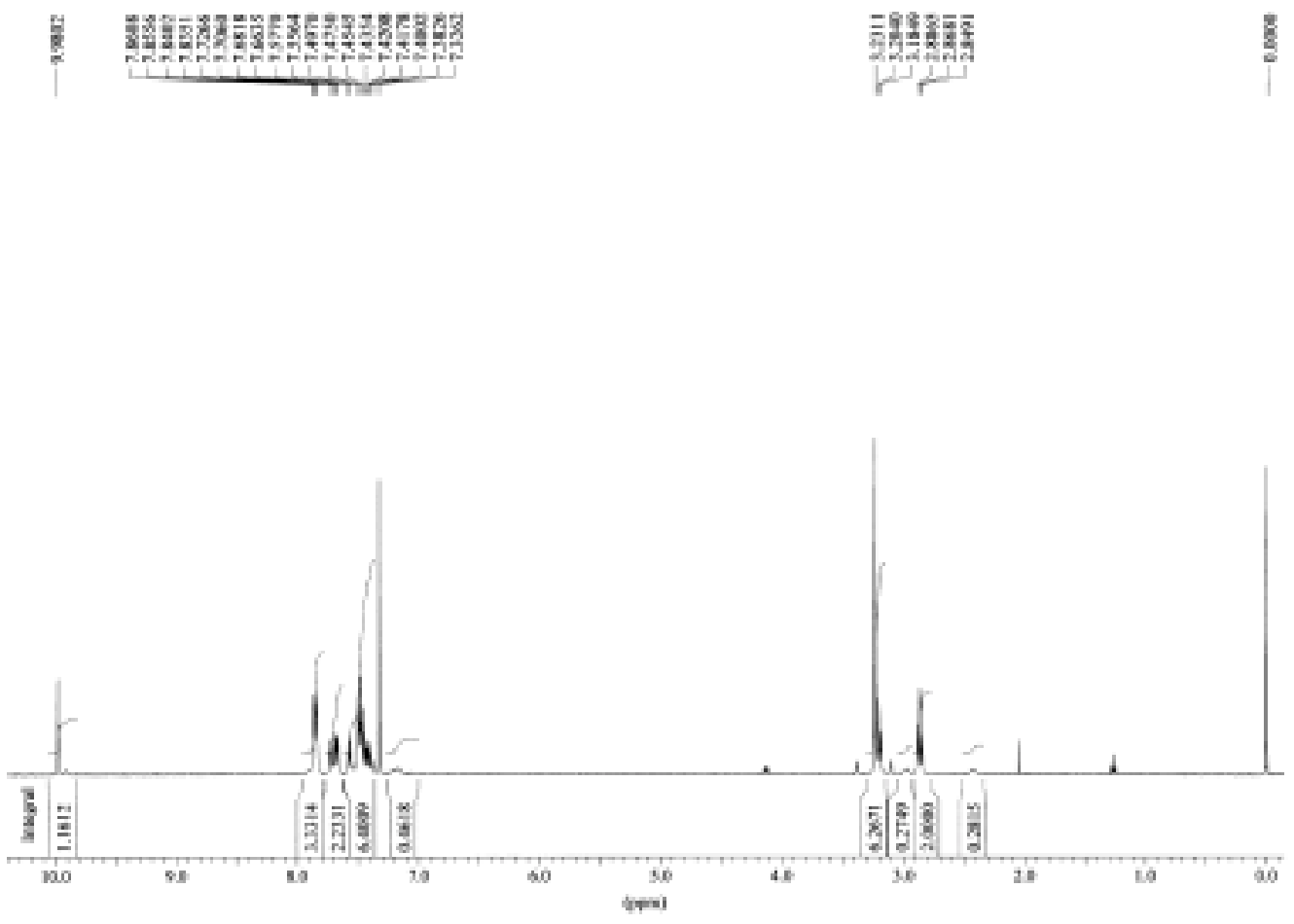
Compound 6, ${ }^{1} \mathrm{H} \mathrm{NMR}\left(400 \mathrm{MHz}, \mathrm{CDCl}_{3}-\mathrm{CD}_{3} \mathrm{OD}\right)$

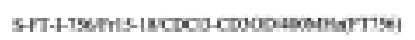
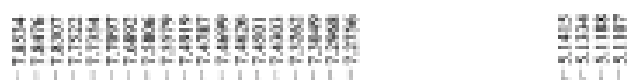

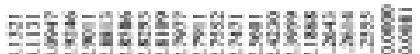

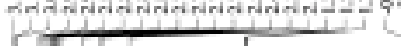

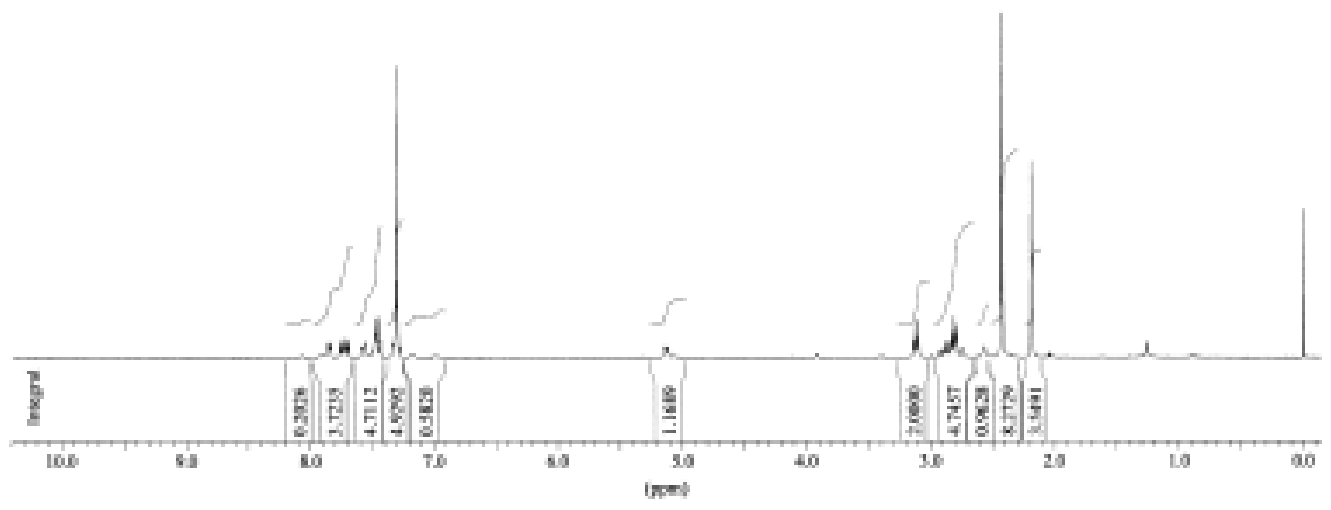

Compound 7, ${ }^{1} \mathrm{H} \mathrm{NMR}\left(400 \mathrm{MHz}, \mathrm{CDCl}_{3}-\mathrm{CD}_{3} \mathrm{OD}\right)$

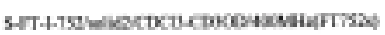

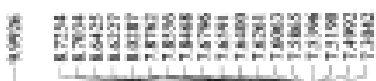

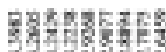

Ir rit

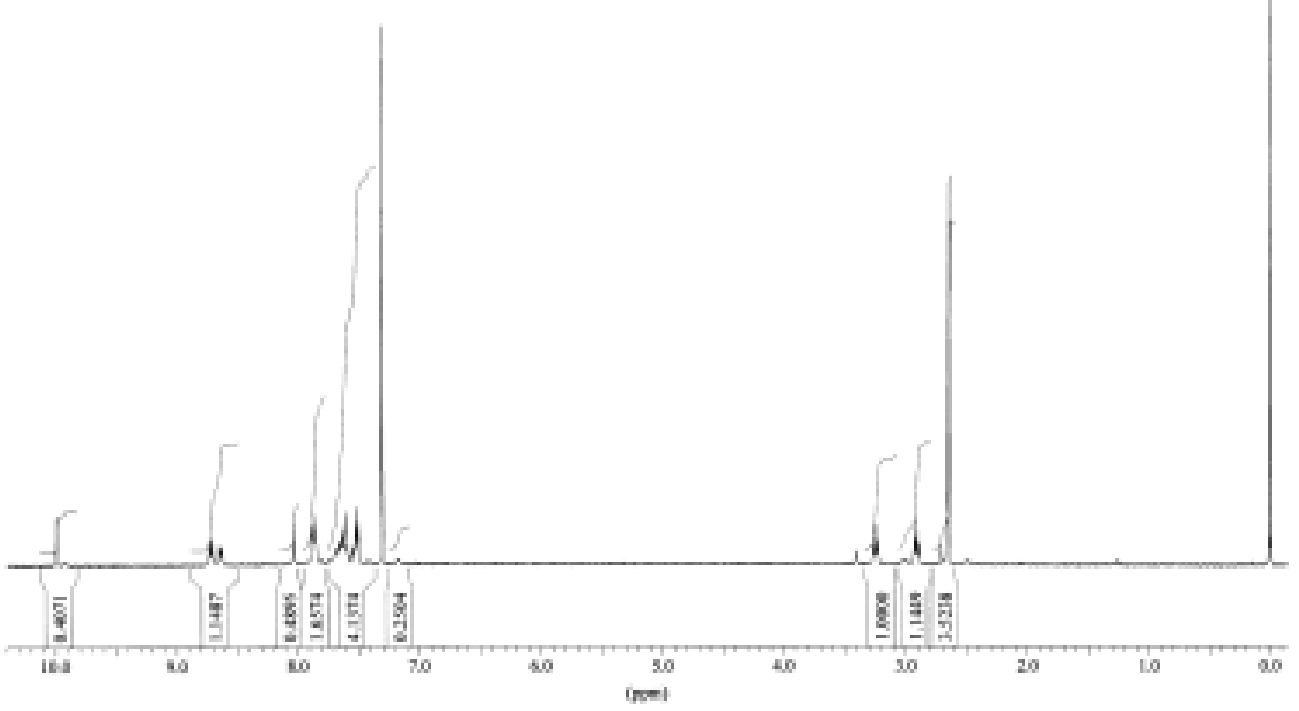


Compound $7,{ }^{13} \mathrm{C}$ NMR $\left(100 \mathrm{MHz}, \mathrm{CDCl}_{3}-\mathrm{CD}_{3} \mathrm{OD}\right)$

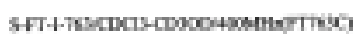

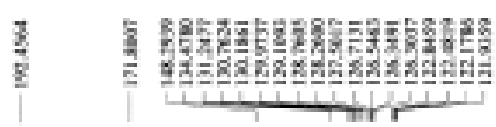

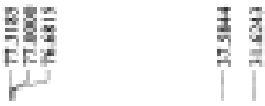

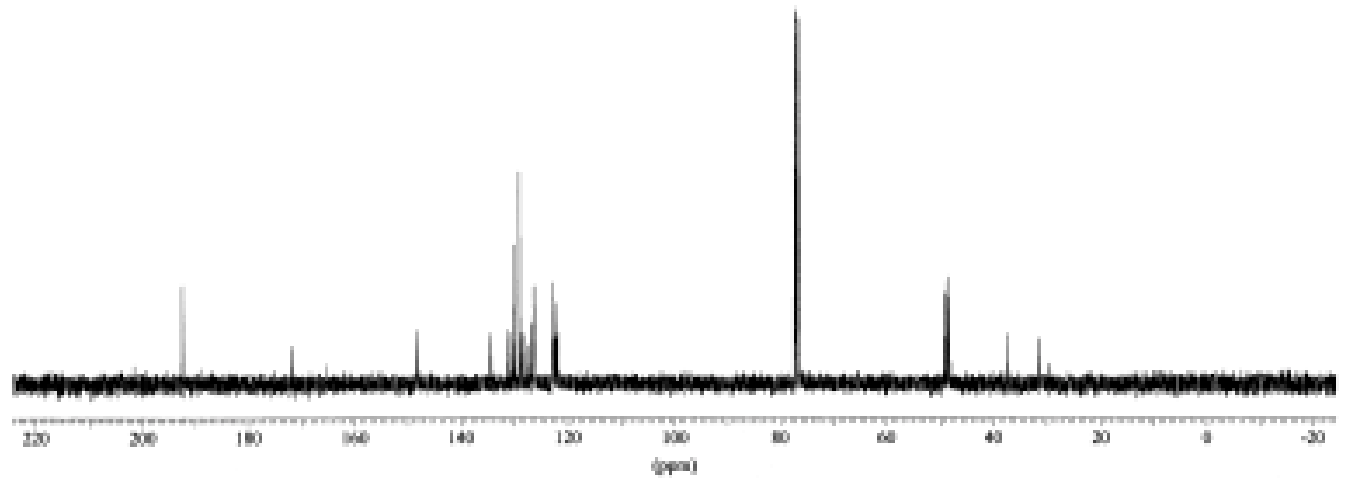

Compound 8, ${ }^{1} \mathrm{H}$ NMR $\left(500 \mathrm{MHz}, \mathrm{CDCl}_{3}\right)$

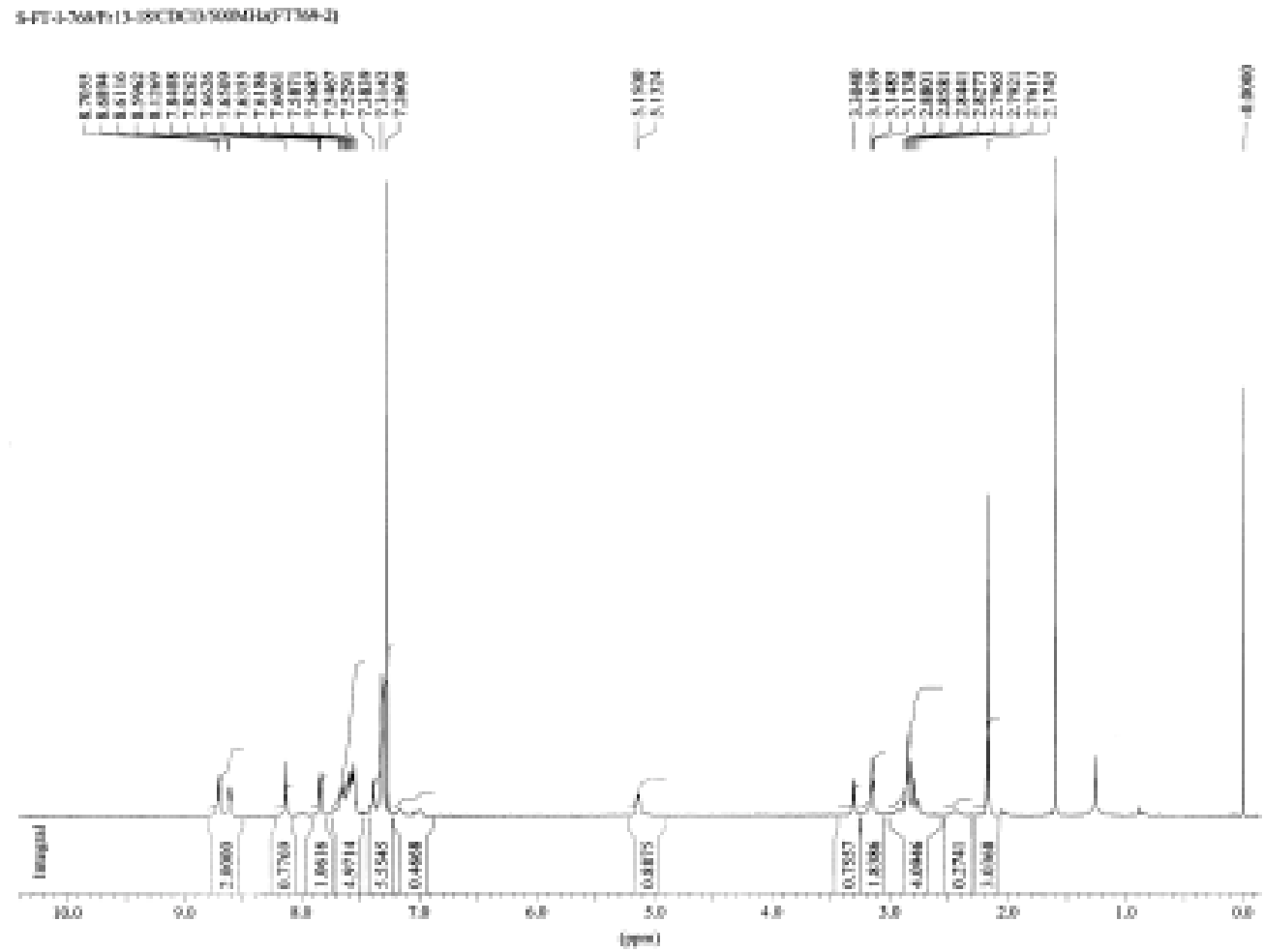


Compound 8, ${ }^{13} \mathrm{C}$ NMR $\left(100 \mathrm{MHz}, \mathrm{CDCl}_{3}\right)$

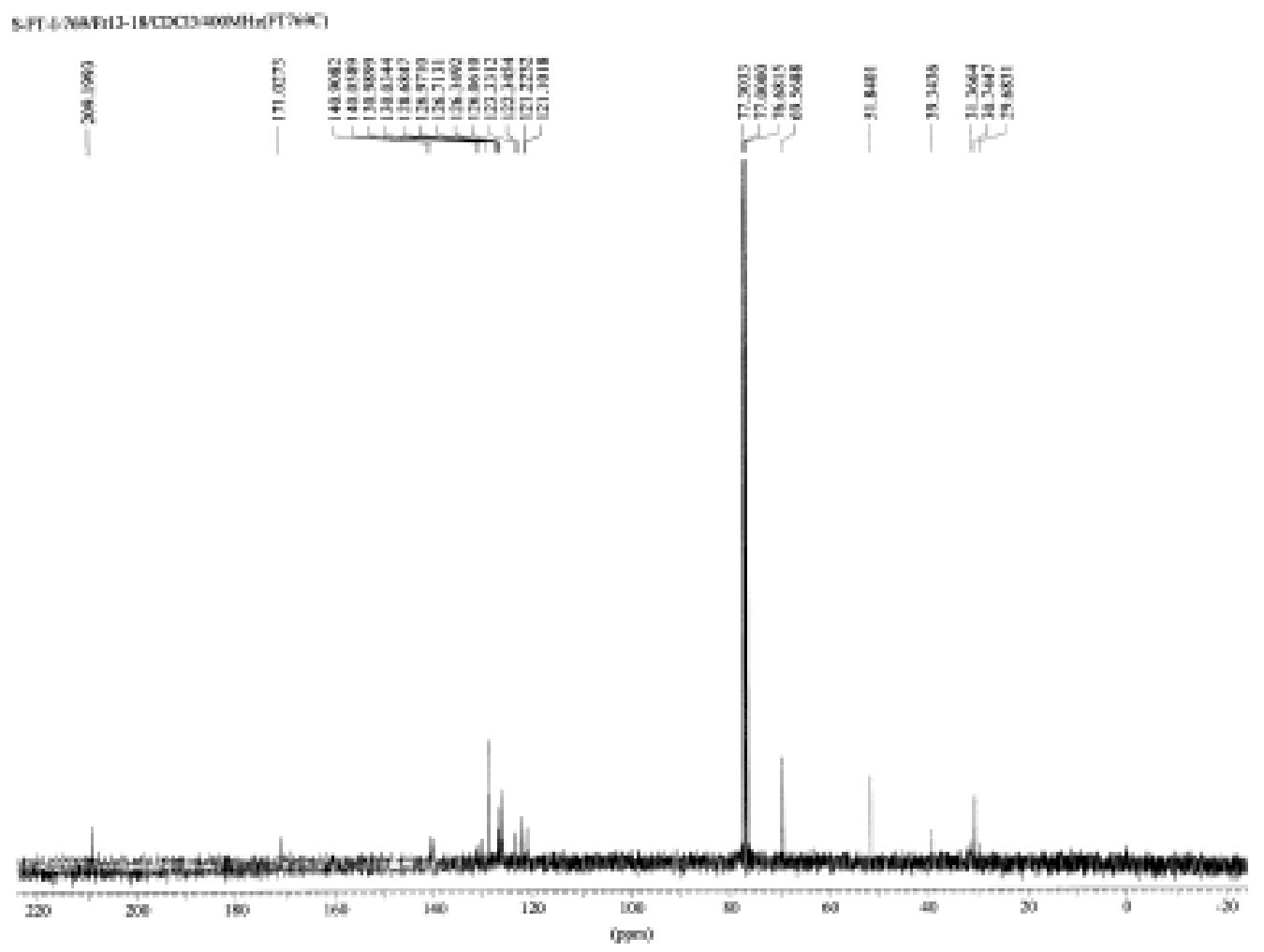

Compound 9, ${ }^{1} \mathrm{H}$ NMR $\left(400 \mathrm{MHz}, \mathrm{CDCl}_{3}-\mathrm{CD}_{3} \mathrm{OD}\right)$
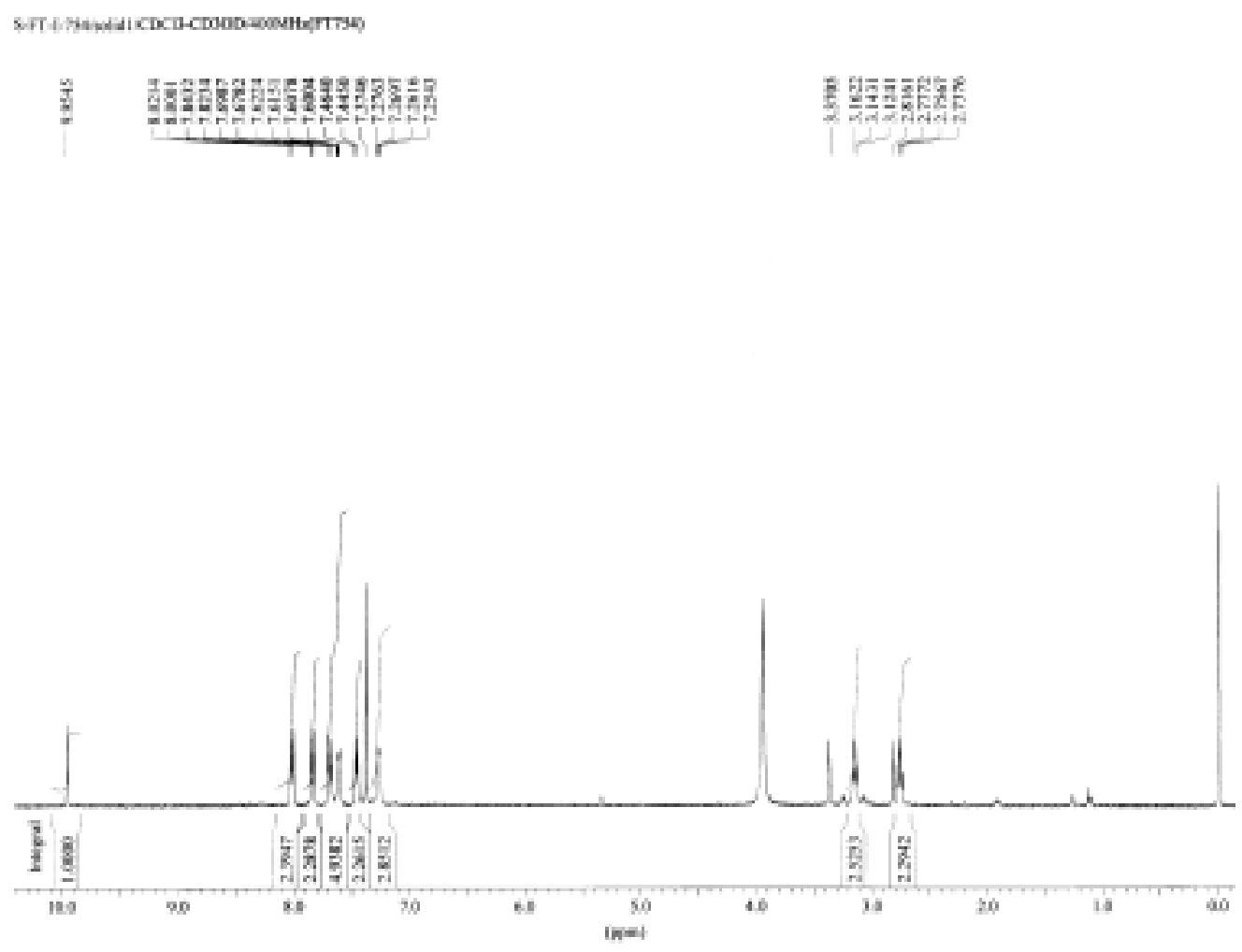
Compound 10, ${ }^{1} \mathrm{H}$ NMR (400MHz, $\left.\mathrm{CDCl}_{3}-\mathrm{CD}_{3} \mathrm{OD}\right)$

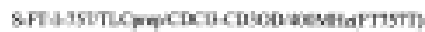
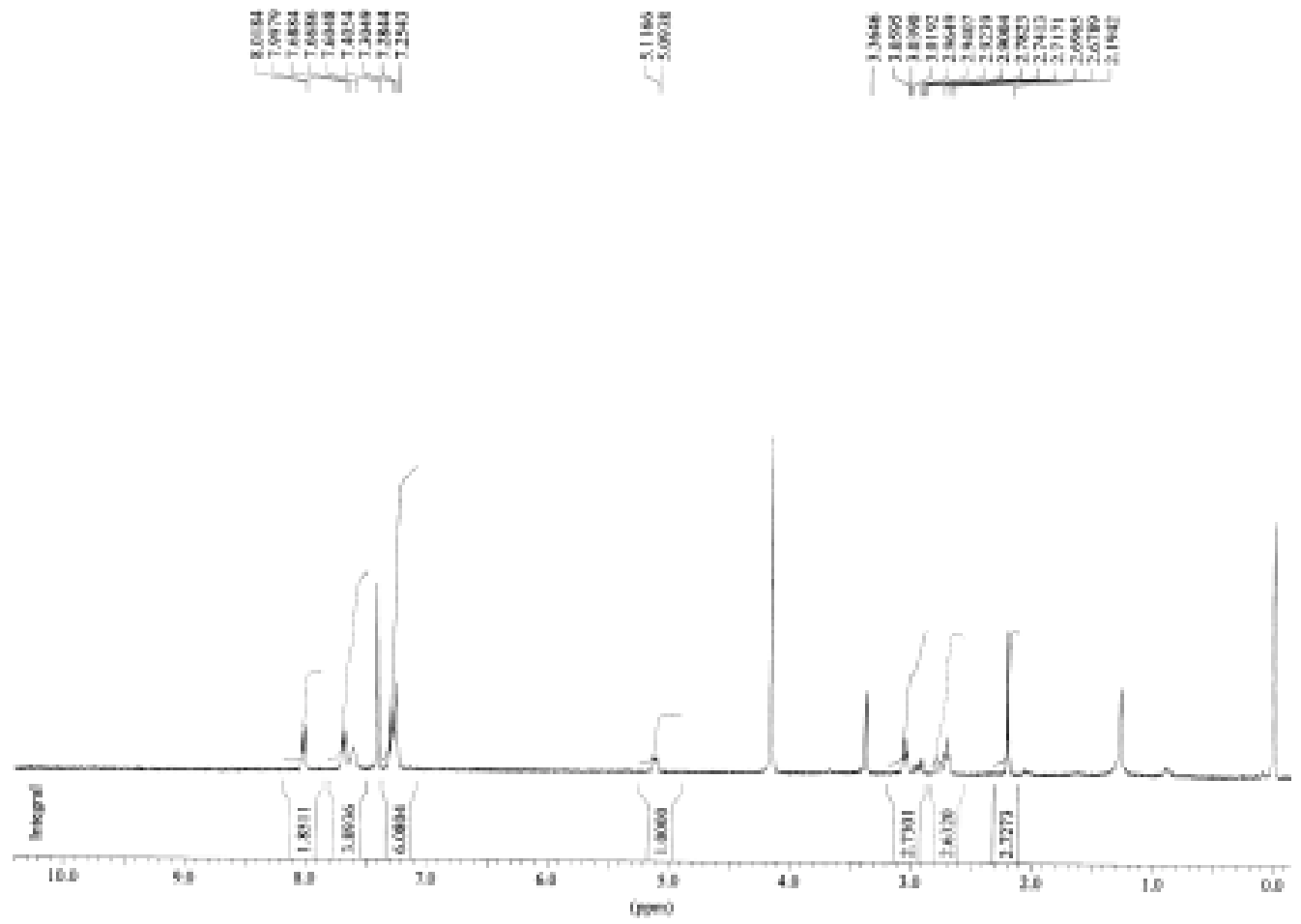

Compound 11, ${ }^{1} \mathrm{H}$ NMR (400MHz, $\left.\mathrm{CDCl}_{3}-\mathrm{CD}_{3} \mathrm{OD}\right)$

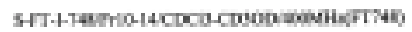

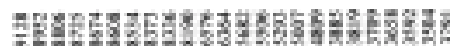
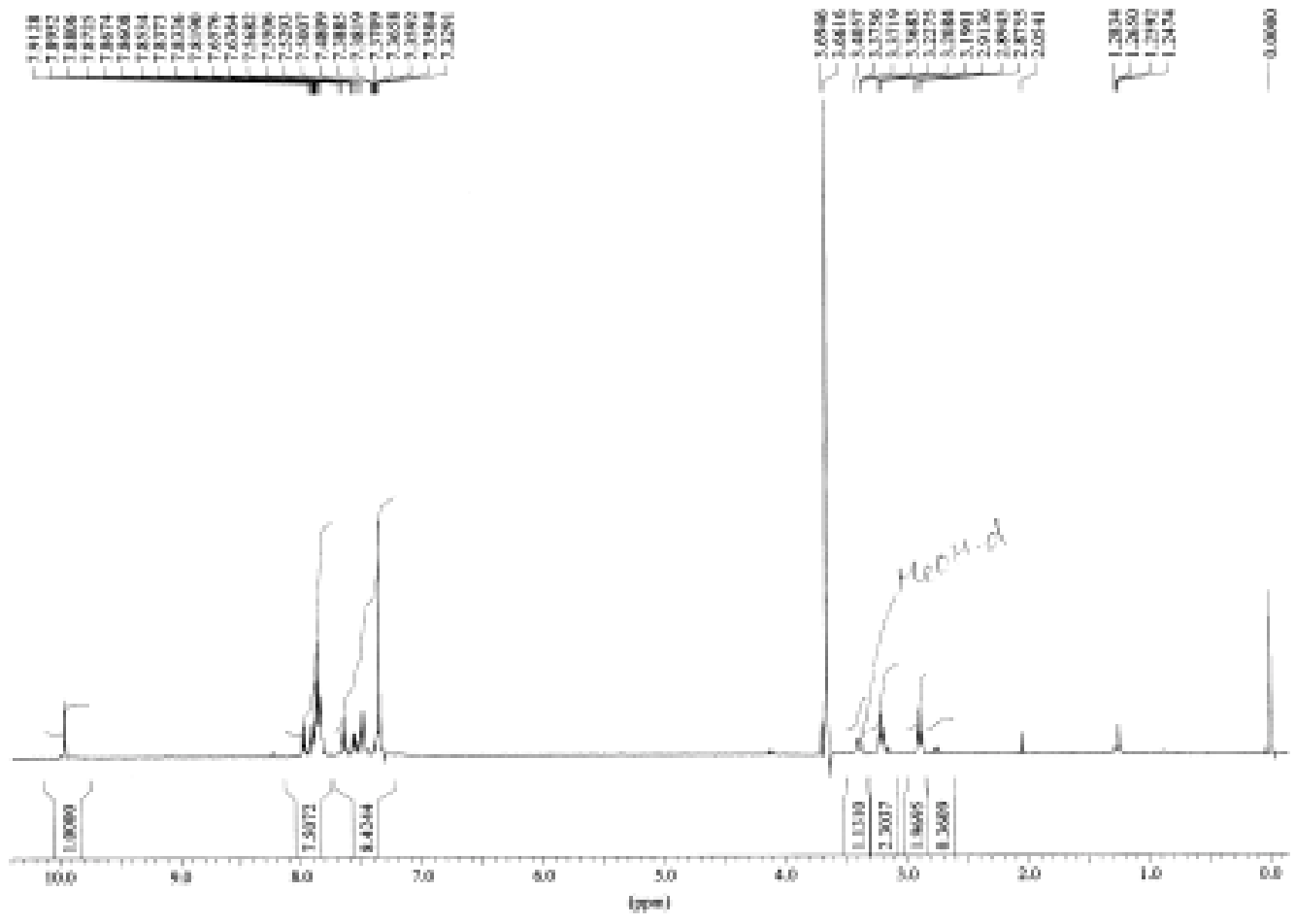
Compound 12, ${ }^{1} \mathrm{H} \mathrm{NMR}\left(400 \mathrm{MHz}, \mathrm{CDCl}_{3}-\mathrm{CD}_{3} \mathrm{OD}\right)$

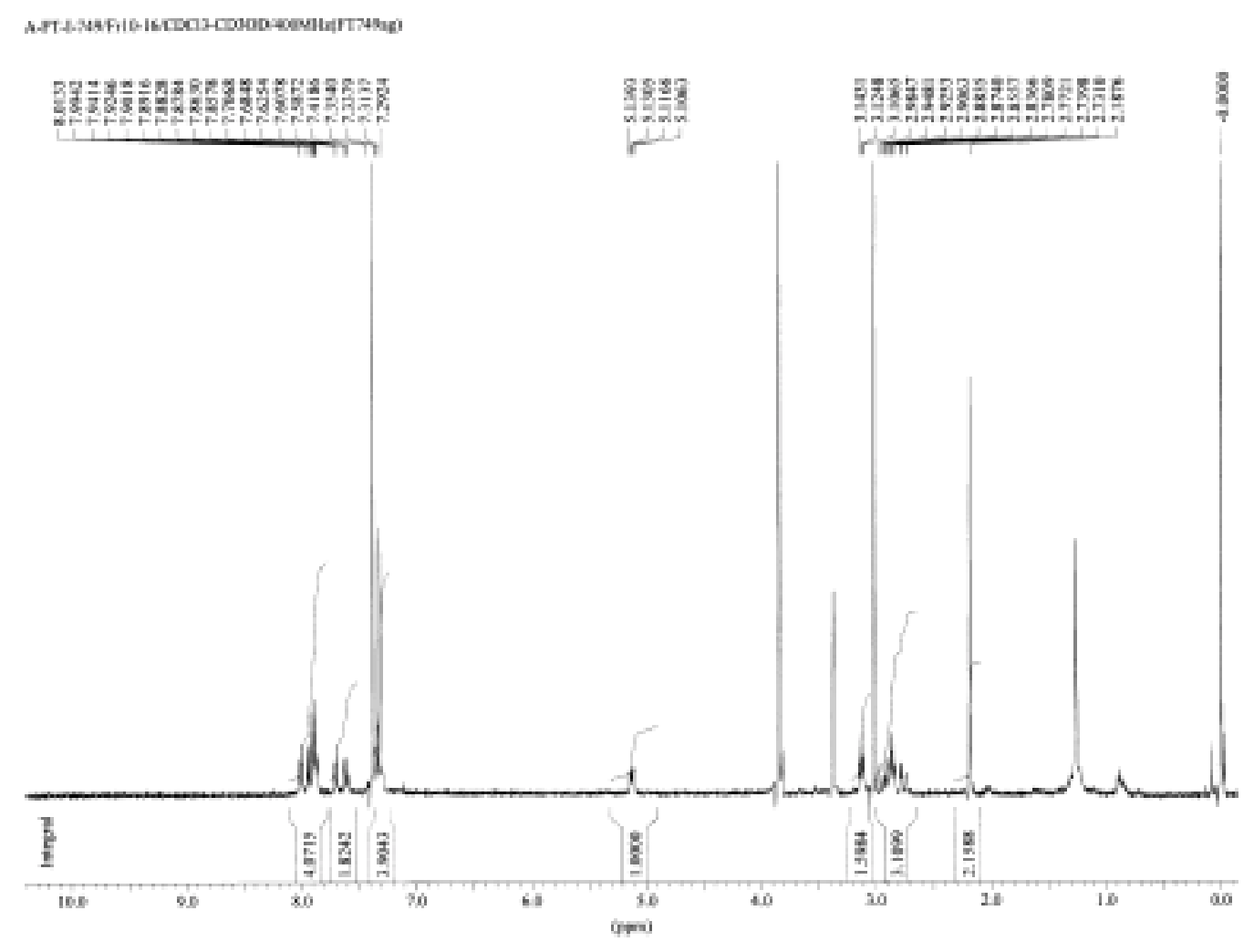

Compound 14, ${ }^{1} \mathrm{H}$ NMR $\left(300 \mathrm{MHz}, \mathrm{CDCl}_{3}\right)$

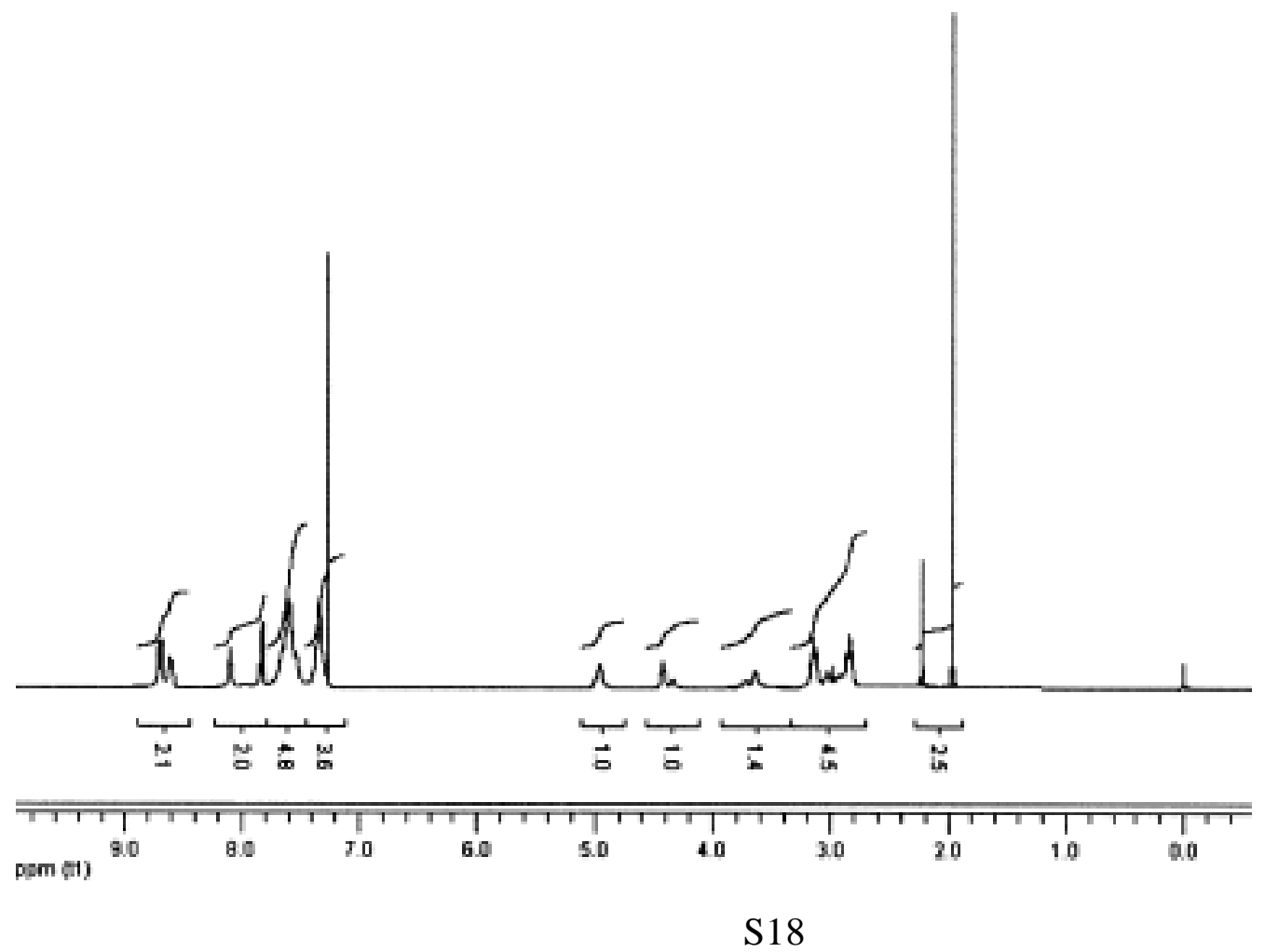


Compound 15, ${ }^{1} \mathrm{H}$ NMR $\left(500 \mathrm{MHz}, \mathrm{CDCl}_{3}\right)$

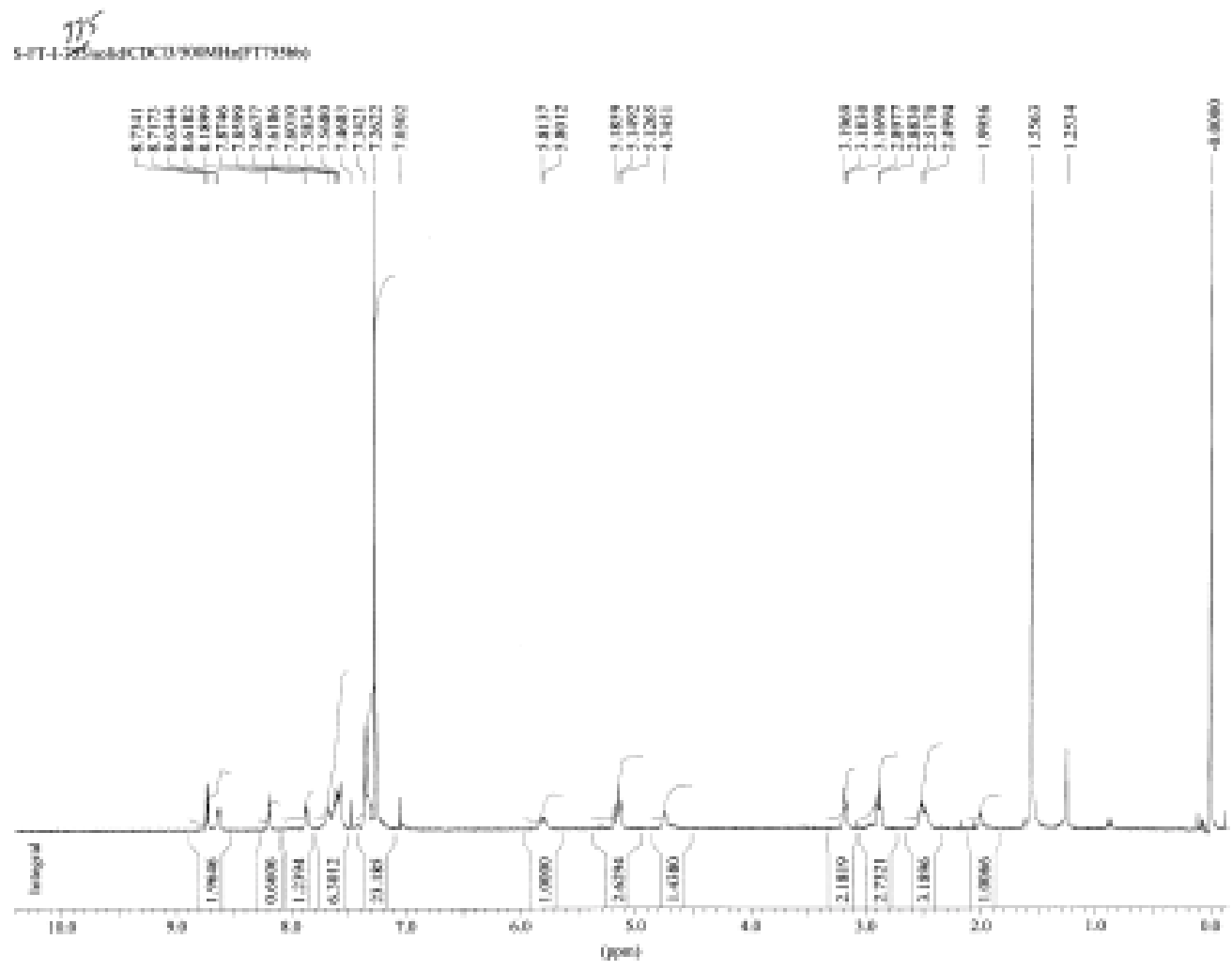

Compound $15,{ }^{13} \mathrm{C}$ NMR $\left(100 \mathrm{MHz}, \mathrm{CDCl}_{3}\right)$

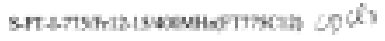
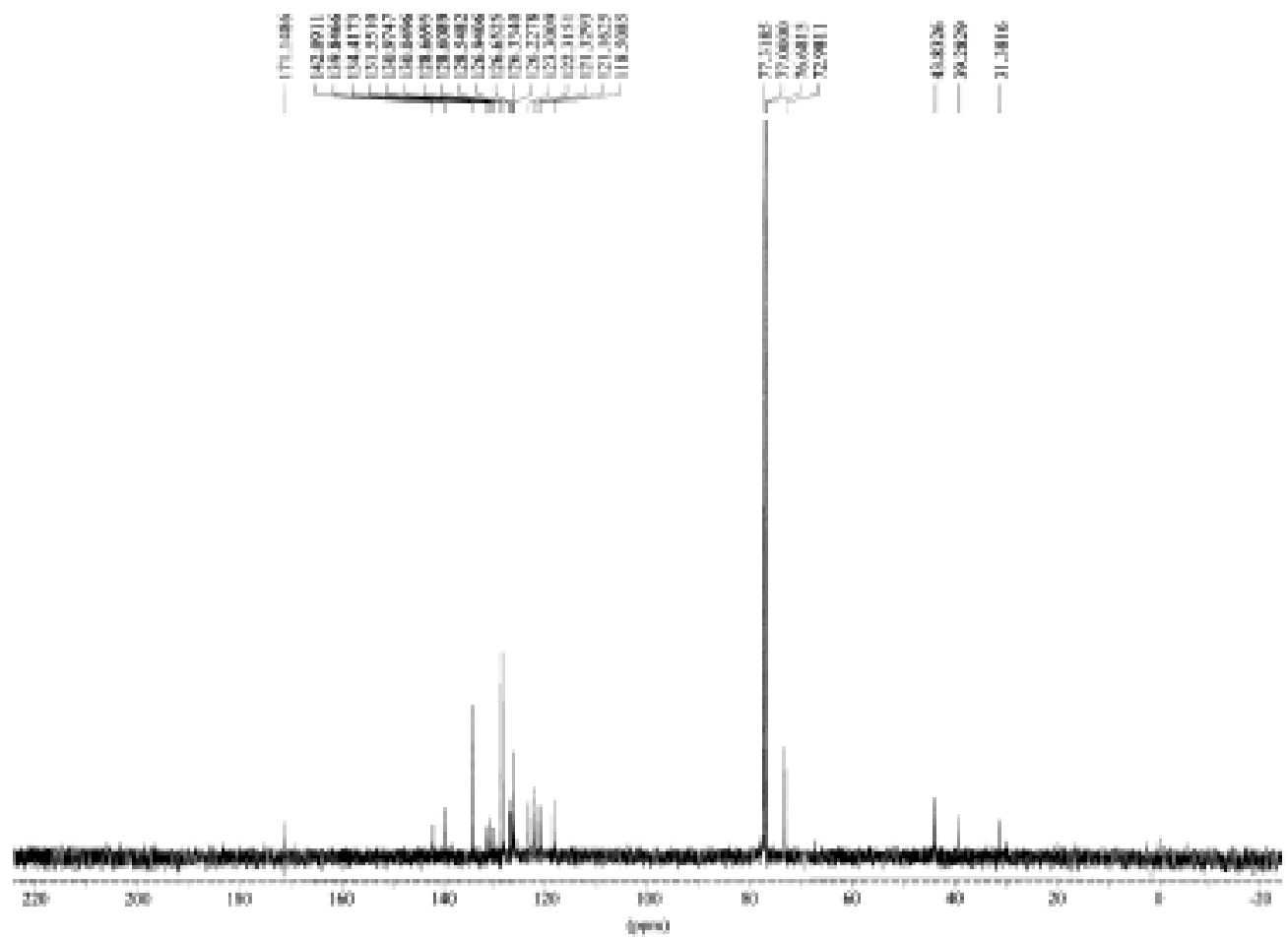
Compound 16, ${ }^{1} \mathrm{H} \mathrm{NMR}\left(500 \mathrm{MHz}, \mathrm{CDCl}_{3}-\mathrm{CD}_{3} \mathrm{OD}\right)$

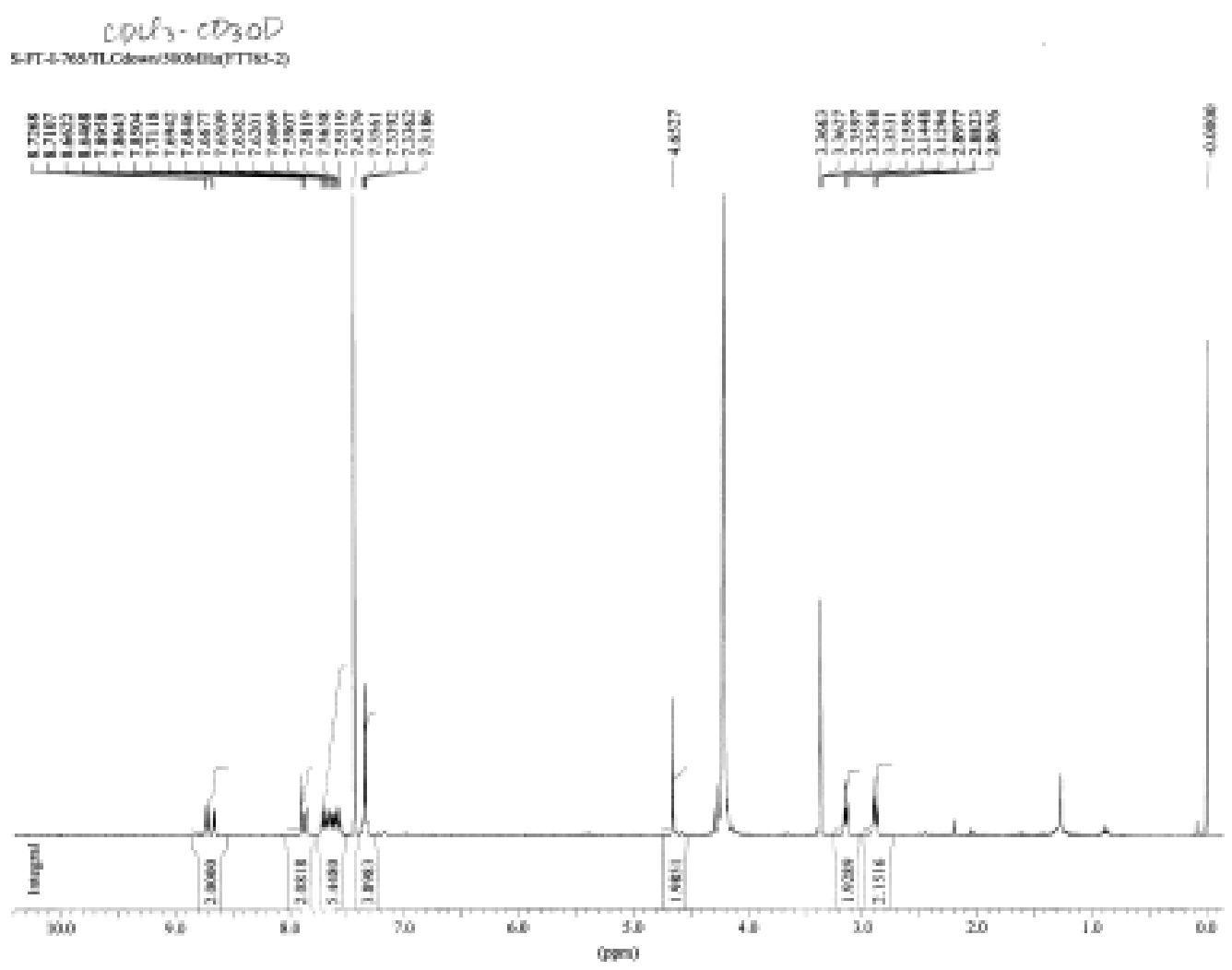

